Classification

Physics Abstracts

$33.80 \mathrm{G}-33.90$

\title{
Coherence effects in the polarization of the light emitted by photofragments
}

\author{
J. Vigué \\ Laboratoire de Spectroscopie Hertzienne, Ecole Normale Supérieure, 24, rue Lhomond, 75231 Paris Cedex 05, \\ France
}

\section{J. A. Beswick}

LURE and Laboratoire de Photophysique Moléculaire, Université de Paris Sud, 91405 Orsay, France

and M. Broyer

Laboratoire de Spectrométrie Ionique et Moléculaire, Université de Lyon I, 69621 Villeurbanne, France

(Reçu le 12 avril 1983, accepté le 18 juillet 1983)

\begin{abstract}
Résumé. - Dans cet article, on présente un traitement quantique général de la photodissociation et de la fluorescence des fragments, pour la photodissociation directe ainsi que pour la photo-prédissociation. Ce traitement prend en compte tous les effets de cohérence possibles provenant des interférences quantiques entre les amplitudes de photodissociation vers différents sous-niveaux magnétiques des fragments. Il en résulte d'importantes différences entre les résultats classiques et quantiques en ce qui concerne le degré de polarisation de la lumière émise par les photofragments. La théorie est appliquée au cas de $\mathrm{Ca}_{2}$ qui constitue un remarquable exemple expérimental des effets de cohérence en photodissociation directe. Pour ce cas, le calcul classique prédit un taux de polarisation de $14 \%$ tandis que dans l'expérience on trouve $64 \%$. La valeur maximum que prédit la théorie quand on tient compte de la cohérence est de $78 \%$. Cette différence entre théorie et expérience est attribuée essentiellement à une dépolarisation par saturation, bien qu'on ne puisse pas exclure d'autres contributions mineures.
\end{abstract}

\begin{abstract}
In this paper a general quantum mechanical treatment of photodissociation and fluorescence from the fragments in the case of direct dissociation as well as in the case of photo-predissociation is presented. This formulation takes into consideration all the possible coherence effects coming from quantum interference between photodissociation amplitudes to different magnetic sublevels of the fragments. This results in large differences between classical and quantum results for the degree of polarization of the light emitted from photofragments. The theory is applied to the case of $\mathrm{Ca}_{2}$ which constitutes a striking experimental example of coherence effects in direct photodissociation. For this case the classical calculation predicts a polarization degree of $14 \%$ while the experiment shows $64 \%$. The maximum theoretical value when coherence is taken into account is $78 \%$. The difference between the experimental result and the theoretical value is attributed to depolarization by saturation, although some other minor contribution may also be present.
\end{abstract}

\section{Introduction.}

The most detailed and important information on photofragmentation dynamics is provided by the analysis of the state of the fragments : angular, velocity and internal state distributions [1]. Such information can be obtained by the use of a variety of techniques including, mass spectrometric detection of products and time of flight measurements, Fourier transform Doppler spectroscopy, laser induced fluorescence, etc.

A particularly interesting situation arises when the fragmentation produces electronically excited fragments which fluoresce. The measurement of this fluorescence gives direct information on the internal state distribution of the fragments. In particular, the polarization of the fluorescence emitted by the fragments yields information concerning the alignment and orientation of the fragments, as well as on the nature of the photon absorption, the dynamics of the half-collision process, etc. This situation is not restricted to photodissociation experiments since similar examples are found in photoionization processes [2] as well as in chemiluminescence reactions [3]. 
The possibility of observing fluorescence polarization from fragments in molecular dissociation processes was proposed by van Brunt and Zare [4]. Defining the degree of polarization by

$$
P=\left(I_{\|}-I_{\perp}\right) /\left(I_{\|}+I_{\perp}\right)
$$

where $I_{\|}$and $I_{\perp}$ are the intensities of the fluorescence with polarization parallel and perpendicular to the polarization of the incident photon, they carried out the calculation in two steps : 1) evaluating the parallel and perpendicular polarization intensities for each possible orientation of the internuclear axis in space; 2) averaging the intensities over the angular distribution of the internuclear axis produced by the photodissociation process.

From their calculation van Brunt and Zare [4] concluded that two necessary conditions for polarization are : (a) an anisotropic angular distribution of dissociation products, $(b)$ a preferential population of the magnetic sublevels of the excited fragments. These two conditions are ordinarily met in most cases. The appearence of an anisotropic spatial distribution of products in the fragmentation of a molecular system is well-known theoretically [5] and demonstrated experimentally for photon as well as for electron, fast ion- and neutral-particle impact [6]. The preferential population of magnetic sublevels of the excited fragments is obtained whenever the dissociation proceeds adiabatically. In that case, for a given molecular state of the $\mathrm{AB}$ molecule there corresponds a state $(\Omega=0)$ or a pair of states $(\Omega= \pm 1$ or $\Omega=$ \pm 2 , etc.) of the separated atoms having fixed values of the magnetic quantum number in the molecular frame of reference.

When the dissociation proceeds fast as compared to the rotational period of the molecule (axial recoil), the van Brunt and Zare's treatment gives

$$
P=\left(3\left\langle\cos ^{2} \gamma\right\rangle-1\right) /\left(\left\langle\cos ^{2} \gamma\right\rangle+3\right)
$$

where $\gamma$ is the angle between the absorbing and emitting transition dipole moments in the molecular frame, and $\langle>$ denotes the average over all possible initial orientations of the molecule. Equation 2 is a well-known result in the theory of luminescence of molecules (not necessarily diatomics) and can be obtained by a simple classical model in terms of absorbing and emitting linear oscillators [7]. According to equation 2 , the degree of polarization should vary between $1 / 2$ and $-1 / 3$.

Polarized fluorescence has been observed from electronically excited diatomic fragments in the photodissociation and predissociation of polyatomic molecules [8]. Rothe, Krause and Düren [9] have observed $-5 \%$ polarized emission of excited $\mathrm{Na}\left({ }^{2} \mathrm{P}_{3 / 2}\right)$ atoms from photodissociation of $\mathrm{Na}_{2}$. The first example of polarized fluorescence from molecular ions produced by photoionization was recently observed in $\mathrm{N}_{2}$ [2].
All these experiments show a degree of polarization within the limits defined by equation 2 .

Interest in the theory of fluorescence polarization in photofragmentation has recently been revived by an experiment on $\mathrm{Ca}_{2}$, where for the first time a large degree of polarization $(64 \%)$ was observed [10]. Furthermore, this result is in contradiction with the predictions of equation 2. Vigué and collaborators [10] explained this surprising result by the consideration of coherence effects between magnetic sublevels of the fragments. The interpretation was the following : the photodissociation of $\mathrm{Ca}_{2}$ with a photon energy of $\sim 3 \mathrm{eV}$ is due to the excitation from the ground $\mathrm{X}^{1} \Sigma_{\mathrm{g}}^{+}$of a ${ }^{1} \Pi_{\mathrm{u}}$ state. This yields after dissociation one ${ }^{1} S_{0}$ atom and one ${ }^{1} P_{1}$ atom, the latter being in a coherent superposition of the magnetic sublevels $m= \pm 1$. The maximum degree of polarization which can be obtained when this coherent superposition is taken into account is $78 \%$.

The calculation presented by Vigué and coll. [10] was performed in the same spirit as of the van Brunt and Zare's paper [4], i.e., by convoluting the spatial orientation of products and the fluorescence intensity. The difference of the results comes from the fact that in the van Brunt and Zare's work the excited atom is assumed to be populated in an incoherent superposition of the $m= \pm 1$ magnetic sublevels and thus resulting in a smaller degree of polarization. It was concluded [10] that coherence effects in photodissociation processes are amenable to experimental evidence and that the case of $\mathrm{Ca}_{2}$ constitutes a striking example of this.

It should be noted at this point that the question considered here is intimately related to the phenomenon of orientation and alignment of atoms produced in photodissociation of molecules. Recently [11], Vasyutinskii has reported a large degree of oriented cesium atoms, produced by photolysis of CsI molecules by circularly polarized light. In a recent theoretical paper [12], he analyses the conditions under which this phenomena may be observed in other molecules. It turns out that these conditions are quite general and they make possible to obtain oriented atoms which many times cannot be obtained by ordinary methods.

The calculations presented by Vigué et al. [10] were performed by neglecting the molecular rotation. It seems important to develop a general quantum mechanical formalism which could include rotational effects as well. Also, it is interesting to study the conditions under which the degree of polarization can be calculated by the van Brunt and Zare's treatment [4], i.e., by convoluting angular distributions and fluorescence from the fragments.

The purpose of this paper is twofold : on one hand we present the general quantum mechanical expressions for photodissociation and fluorescence from the fragments in the case of direct dissociation as well as in the case of predissociation, and on the 
other hand, we establish the conditions under which the complete quantum mechanical treatment reduces to that of van Brunt and Zare [4]. Finally, we apply our general expressions to the case of $\mathrm{Ca}_{2}$, studied experimentally by Vigué, Grangier, Roger and Aspect [10], and we discuss several possible mechanisms for depolarization.

\section{Quantum mechanical formalism for the case of direct photodissociation.}

2.1 General treatment. - Consider a quantum mechanical material system described by the Hamiltonian $H_{\mathrm{M}}$, and three sets of eigenstates : $a$ ) a set of initial states $\left|\alpha_{i}\right\rangle$ described by a collective quantum number $\left.\alpha_{i} ; b\right)$ a set of excited states $\left|\alpha_{d}\right\rangle$ which can be populated by photon absorption from the initial states; $c$ ) and a set of final states $\left|\alpha_{\mathrm{f}}\right\rangle$ obtained by emission of a photon from the excited states $\left|\alpha_{d}\right\rangle$. Since we shall deal with weak electromagnetic fields, we consider only two types of eigenstates of the radiation field Hamiltonian $H_{\mathrm{R}}$ : the zero photon state $|0\rangle$ and the one photon states $|\mathbf{k}, \mathbf{e}\rangle$, where $\mathbf{k}$ is the wavevector associated to a photon with energy $\hbar c k$ and $\mathbf{e}$ being its polarization vector.

We are interested in the calculation of the cross section for the process :

$$
\left|\alpha_{\mathrm{i}}\right\rangle \stackrel{\mathbf{k}_{\mathrm{i}} \mathbf{e}_{\mathrm{i}}}{\longrightarrow}\left|\alpha_{\mathrm{d}}\right\rangle \stackrel{\mathbf{k}_{\mathbf{f}} \mathbf{e}_{\mathrm{f}}}{\longrightarrow}\left|\alpha_{\mathrm{f}}\right\rangle
$$

corresponding to the excitation of the system with a photon $\mathbf{k}_{\mathrm{i}} \mathbf{e}_{\mathrm{i}}$, and deexcitation by a photon $\mathbf{k}_{\mathrm{f}} \mathbf{e}_{\mathrm{f}}$. The relevant eigenstates for the matter plus radiation field zero-order Hamiltonian $H_{0}=H_{\mathrm{M}}+H_{\mathrm{R}}$, will then be : $\left|\alpha_{i} ; \mathbf{k}_{\mathrm{i}} \mathbf{e}_{\mathrm{i}}\right\rangle ;\left|\alpha_{\mathrm{d}} ; 0\right\rangle ;\left|\alpha_{\mathrm{f}} ; \mathbf{k}_{\mathrm{f}} \mathbf{e}_{\mathrm{f}}\right\rangle$. These zero-order states are coupled by the field-matter interaction operator $H_{\text {int }}$

The partial cross section for absorption of a photon $\mathbf{k}_{\mathrm{i}} \mathbf{e}_{\mathrm{i}}$, from the specified initial state $\left|\alpha_{\mathrm{i}}\right\rangle$, and emission of photons $\mathbf{k}_{\mathrm{f}} \mathbf{e}_{\mathrm{f}}$ in the solid angle $\hat{\omega}_{\mathrm{f}}$, is given by

$$
\begin{aligned}
\frac{\mathrm{d} \sigma}{\mathrm{d} \hat{\omega}_{\mathrm{f}}}=\sum_{\alpha_{\mathrm{f}}} 2 \pi k_{\mathrm{f}}^{2}(\hbar c)^{-2} \times \\
\quad \times\left|\left\langle\alpha_{\mathrm{i}} ; \mathbf{k}_{\mathrm{i}} \mathbf{e}_{\mathrm{i}}|T(E)| \alpha_{\mathrm{f}} ; \mathbf{k}_{\mathrm{f}} \mathbf{e}_{\mathrm{f}}\right\rangle\right|^{2}
\end{aligned}
$$

where the calculation is performed on the energy shell, i.e., $E=\varepsilon_{\mathrm{f}}+\hbar c k_{\mathrm{f}}=E_{\mathrm{i}}+\hbar c k_{\mathrm{i}}$. In writing equation 4 we have chosen $k$-normalized photon states, i.e., $\left\langle\mathbf{k} \mid \mathbf{k}^{\prime}\right\rangle=\delta\left(\mathbf{k}-\mathbf{k}^{\prime}\right)$ and energy normalized continuum states. This implies that the sum over $\alpha_{\mathrm{f}}$ includes an integral over $\varepsilon_{\mathrm{f}}$. The operator $T(E)$ in equation 4 is the usual transition operator defined by :

$$
T(E)=H_{\mathrm{int}}+H_{\mathrm{int}} G^{+}(E) H_{\mathrm{int}}
$$

where $G^{+}(E)$ is the resolvent operator

$$
G^{+}(E)=\lim _{\eta \rightarrow 0}(E-H+i \eta)^{-1} .
$$

From (4) to (6), it can be shown that (cf. appendix D) :

$\frac{\mathrm{d} \sigma}{\mathrm{d} \hat{\omega}_{\mathrm{f}}}=\sum_{\alpha_{\mathrm{f}}} 2 \pi k_{\mathrm{f}}^{2}(\hbar c)^{-2}\left|\sum_{\alpha_{\mathrm{d}}}\left\langle\alpha_{\mathrm{i}} ; \mathbf{k}_{\mathrm{i}} \mathbf{e}_{\mathrm{i}}\left|H_{\mathrm{int}}\right| \alpha_{\mathrm{d}} ; 0\right\rangle\left(E-E_{\mathrm{d}}+i \Gamma_{\mathrm{d}}\right)^{-1}\left\langle\alpha_{\mathrm{d}} ; 0\left|H_{\mathrm{int}}\right| \alpha_{\mathrm{f}} ; \mathbf{k}_{\mathrm{f}} ; \mathbf{k}_{\mathrm{f}} \mathbf{e}_{\mathrm{f}}\right\rangle\right|^{2}$

where $\Gamma_{\mathrm{d}}$ is the fragment radiative linewidth. To proceed further we need now to specify the matrix elements of $H_{\text {int }}$. They can be written, in the dipole approximation,

$$
\begin{aligned}
& \left\langle\alpha_{\mathrm{i}} ; \mathbf{k}_{\mathrm{i}} \mathbf{e}_{\mathrm{i}}\left|H_{\text {int }}\right| \alpha_{\mathrm{d}} ; 0\right\rangle=\left(\hbar c k_{\mathrm{i}}\right)^{1 / 2}\left\langle\alpha_{\mathrm{i}}\right| \text { D. } \mathbf{e}_{\mathrm{i}}\left|\alpha_{\mathrm{d}}\right\rangle \\
& \left\langle\alpha_{\mathrm{d}} ; 0\left|H_{\text {int }}\right| \alpha_{\mathrm{f}} ; \mathbf{k}_{\mathrm{f}} \mathbf{e}_{\mathrm{f}}\right\rangle=\left(\hbar c k_{\mathrm{f}}\right)^{1 / 2}\left\langle\alpha_{\mathrm{d}}\right| \text { D. } \mathrm{e}_{\mathrm{f}}^{*}\left|\alpha_{\mathrm{f}}\right\rangle
\end{aligned}
$$

where $\mathbf{D}$ is the electronic transition dipole operator. We shall now specify explicitly the states $\left|\alpha_{i}\right\rangle,\left|\alpha_{d}\right\rangle$ and $\left|\alpha_{\mathrm{f}}\right\rangle$. We denote by $\left|\Omega_{\mathrm{i}} v_{\mathrm{i}} J_{\mathrm{i}} M_{\mathrm{i}}\right\rangle$ the initial state, characterized by the total angular momentum quantum number $J_{\mathrm{i}}$, its projection $M_{\mathrm{i}}$ in the laboratory $z$-axis, its projection $\Omega_{\mathrm{i}}$ in the molecular axis, and a vibrational quantum number $v_{\mathrm{i}}$. We thus assume implicitly the wavefunction to be described in the $(c)$ Hund's case coupling scheme. Similarly we write $\left|\Omega_{\mathrm{d}} \varepsilon_{\mathrm{d}} J_{\mathrm{d}} M_{\mathrm{d}}\right\rangle$ and $\left|\Omega_{\mathrm{f}} \varepsilon_{\mathrm{f}} J_{\mathrm{f}} M_{\mathrm{f}}\right\rangle$ for the intermediate and final wavefunctions with $\varepsilon_{\mathrm{d}}$ and $\varepsilon_{\mathrm{f}}$ being the energy of the fragments (electronic plus relative kinetic energy). Denoting by $\mathbf{R}$ the internuclear distance and by $\mathbf{r}$ the ensemble of electronic coordinates, we then have

$$
\left\langle\mathbf{R}, \mathbf{r} \mid \Omega_{\mathrm{i}} v_{\mathrm{i}} J_{\mathrm{i}} M_{\mathrm{i}}\right\rangle=\phi_{\Omega_{\mathrm{i}}}(\mathbf{r} ; \mathbf{R}) \chi_{v_{\mathrm{i}} J_{\mathrm{i}} \Omega_{\mathrm{i}}}(R)\left(\frac{2 J_{\mathrm{i}}+1}{4 \pi}\right)^{1 / 2} \mathfrak{D}_{M_{\mathrm{i}} \Omega_{\mathrm{i}}}^{J *}(\varphi, \theta, 0)
$$

where $\theta, \varphi$ are the polar angles specifying the orientation of the molecular axis with respect to the laboratory system of coordinates. Similar expressions hold for the intermediate and final states. It should be noticed at this point that the appropriate molecular wavefunctions are those with definite parity :

$$
\left|\Omega_{\mathrm{i}}, p_{\mathrm{i}}\right\rangle=(2)^{-1 / 2}\left(\left|\Omega_{\mathrm{i}}\right\rangle+(-)^{p_{\mathrm{i}}}\left|-\Omega_{\mathrm{i}}\right\rangle\right) ; \quad p_{\mathrm{i}}=0,1 .
$$

However, in virtue of the closure relation, the sum over the intermediate states $\left|\Omega_{\mathrm{d}}\right\rangle$ can be performed in either one of the basis sets. In addition, in many cases $\Omega=0$ for the initial and final states and those are already parity defined states. For simplicity we shall present here the treatment with wavefunctions defined as in equation 9 . The consideration of parity defined wavefunctions is trivial but they complicate considerably the expressions. 
Using equation 9 we obtain :

$$
\begin{aligned}
& \langle\Omega v J M| \text { D.e }\left|\Omega^{\prime} v^{\prime} J^{\prime} M^{\prime}\right\rangle=\sum_{p, m}(-1)^{p}(e)_{p}^{1}\left\langle\Omega v J\left|(D)_{m}^{1}\right| \Omega^{\prime} v^{\prime} J^{\prime}\right\rangle \times \\
& (2 J+1)^{1 / 2}\left(2 J^{\prime}+1\right)^{1 / 2}(-1)^{J-M}\left(\begin{array}{ccc}
J & 1 & J^{\prime} \\
-M & -p & M^{\prime}
\end{array}\right)(-1)^{J-\Omega}\left(\begin{array}{ccc}
J & 1 & J^{\prime} \\
-\Omega & m & \Omega^{\prime}
\end{array}\right)
\end{aligned}
$$

where $(e)_{p}^{1}$ are the spherical components, in the laboratory frame, of the polarization vector, i.e. :

$$
(e)_{0}^{1}=\mathbf{e} . \hat{z} ; \quad(e)_{ \pm 1}^{1}=\mp(z)^{-1 / 2}(\mathbf{e} . \hat{x} \pm i \mathbf{e} . \hat{y})
$$

while $(D)_{m}^{1}$ are the corresponding spherical components of the electronic dipole operator in the molecular frame. Using equation 11 into (8) and the result into (7) we obtain the partial cross section from a specified initial state $\left|\Omega_{\mathrm{i}} v_{\mathrm{i}} J_{\mathrm{i}} M_{\mathrm{i}}\right\rangle$ to all final states $\left|\Omega_{\mathrm{f}} \varepsilon_{\mathrm{f}} J_{\mathrm{f}} M_{\mathrm{f}}\right\rangle$. In general however, the initial state of the molecule is randomly oriented and therefore we sum over $M_{\mathrm{i}}$ with a $\left(2 J_{\mathrm{i}}+1\right)^{-1}$ weight factor. The summation over the magnetic quantum numbers $\boldsymbol{M}_{\mathrm{i}}, \boldsymbol{M}_{\mathrm{d}}, \boldsymbol{M}_{\mathrm{f}}$ can be easily performed (see Appendix A) with the result :

$$
\begin{aligned}
& \frac{\mathrm{d} \sigma}{\mathrm{d} \hat{\omega}_{\mathrm{f}}}=2 \pi k_{\mathrm{i}} \int \mathrm{d} \varepsilon_{\mathrm{f}} k_{\mathrm{f}}^{3} \sum_{J_{\mathrm{f}}} \sum_{K=0}^{2} \mathscr{F}_{K} \mid \int \mathrm{d} \varepsilon_{\mathrm{d}} \sum_{\Omega_{\mathrm{d}} J_{\mathrm{d}}}\left(E-\varepsilon_{\mathrm{d}}+i \Gamma_{\mathrm{d}}\right)^{-1}\left\langle\Omega_{\mathrm{i}} v_{\mathrm{i}} J_{\mathrm{i}}\left|(D)_{m}^{1}\right| \Omega_{\mathrm{d}} \varepsilon_{\mathrm{d}} J_{\mathrm{d}}\right\rangle \\
& \left\langle\Omega_{\mathrm{d}} \varepsilon_{\mathrm{d}} J_{\mathrm{d}}\left|(D)_{n}^{1}\right| \Omega_{\mathrm{f}} \varepsilon_{\mathrm{f}} J_{\mathrm{f}}\right\rangle\left(2 J_{\mathrm{f}}+1\right)^{1 / 2}(-1)^{2 J_{\mathrm{d}}} \\
& \left(2 J_{\mathrm{d}}+1\right)\left\{\begin{array}{ccc}
1 & 1 & K \\
J_{\mathrm{f}} & J_{\mathrm{i}} & J_{\mathrm{d}}
\end{array}\right\}\left(\begin{array}{ccc}
J_{\mathrm{i}} & 1 & J_{\mathrm{d}} \\
-\Omega_{\mathrm{i}} & m & \Omega_{\mathrm{d}}
\end{array}\right) \\
& \left.(-1)^{J_{\mathrm{d}}-\Omega_{\mathrm{d}}}\left(\begin{array}{ccc}
J_{\mathrm{d}} & 1 & J_{\mathrm{f}} \\
-\Omega_{\mathrm{d}} & n & \Omega_{\mathrm{f}}
\end{array}\right)\right|^{2} ; \quad \begin{array}{l}
m=\Omega_{\mathrm{i}}-\Omega_{\mathrm{d}} \\
n=\Omega_{\mathrm{d}}-\Omega_{\mathrm{f}}
\end{array} \\
& \mathscr{F}_{K}=(2 K+1) \sum_{Q}\left|\mathcal{F}_{K Q}\right|^{2} \\
& \mathcal{F}_{K Q}=\sum_{p, q}\left(\mathbf{e}_{\mathrm{i}}\right)_{p}^{1}\left(\mathbf{e}_{\mathrm{f}}^{*}\right)_{q}^{1}\left(\begin{array}{lll}
1 & 1 & K \\
p & q & Q
\end{array}\right) .
\end{aligned}
$$

All the information concerning the initial and final polarizations is included in the $\mathscr{F}_{0}, \mathscr{F}_{1}$, and $\mathcal{F}_{2}$ terms. They are tabulated in table I for different experimental conditions. Notice that $\sum_{K} \mathfrak{F}_{K}=1$. This is easy to show
from the normalization of the $3-j$ symbols.

In the context of the Franck-Condon approximation for the matrix elements $\left\langle\Omega_{\mathrm{d}} \varepsilon_{\mathrm{d}} J_{\mathrm{d}}\left|(\mathrm{D})_{n}^{1}\right| \Omega_{\mathrm{f}} \varepsilon_{\mathrm{f}} J_{\mathrm{f}}\right\rangle$, equation 13 can be simplified even further. Writing

$$
\left\langle\Omega_{\mathrm{d}} \varepsilon_{\mathrm{d}} J_{\mathrm{d}}\left|(D)_{n}^{1}\right| \Omega_{\mathrm{f}} J_{\mathrm{f}} \varepsilon_{\mathrm{f}}\right\rangle=\left\langle\chi_{\Omega_{\mathrm{d}} \varepsilon_{\mathrm{d}} J_{\mathrm{d}}} \mid \chi_{\Omega_{\mathrm{f}} \varepsilon_{\mathrm{f}} J_{\mathrm{f}}}\right\rangle \times\left\langle\Omega_{\mathrm{d}}\left|(D)_{n}^{1}\right| \Omega_{\mathrm{f}}\right\rangle
$$

the sums over $\varepsilon_{\mathrm{d}}$ and $\varepsilon_{\mathrm{f}}$ can be performed explicity (cf. appendix C). Since the wavefunctions $\chi_{\Omega_{\mathrm{d} \varepsilon_{\mathrm{d}} J_{\mathrm{d}}}}$ and $\chi_{\Omega_{\mathrm{f}} \varepsilon_{\mathrm{f}} J_{\mathrm{f}}}$ extend over a very large region of the internuclear distance, a question raises whether the Franck-Condon approximation is valid or not. In fact, the vibrational overlap can be divided into two regions : i) an inner region in which the transition dipole moment varies from its molecular value, to the purely atomic value at some distance $R_{0}$; ii) an outer region from $R=R_{0}$ to $R=\infty$, where the transition dipole moment is constant and equal to the atomic transition moment. We shall assume that the contribution of the inner region is negligible compared to that of outer region (this second contribution is important only if $\varepsilon_{\mathrm{d}} \sim \varepsilon_{\mathrm{f}}$ ). Therefore the Franck-Condon approximation is valid with $\left\langle\Omega_{\mathrm{d}}\left|(D)_{n}^{1}\right| \Omega_{\mathrm{f}}\right\rangle$ being the atomic transition dipole moment. This approximation is equivalent to assume that the dissociation time is short compared with the radiative lifetime. This is true in most cases.

Using the results of appendix $\mathrm{C}$ we have finally :

$$
\begin{aligned}
\frac{\mathrm{d} \sigma}{\mathrm{d} \hat{\omega}_{\mathrm{f}}} & =2 \pi^{2} k_{\mathrm{i}} k_{\mathrm{f}}^{3} \Gamma_{\mathrm{d}}^{-1} \sum_{J_{\mathrm{f}}} \sum_{K} \mathcal{F}_{K} \mid \sum_{\Omega_{\mathrm{d}} J_{\mathrm{d}}}\left\langle\Omega_{\mathrm{i}} v_{\mathrm{i}} J_{\mathrm{i}}\left|(D)_{m}^{1}\right| \Omega_{\mathrm{d}} \varepsilon_{\mathrm{d}} J_{\mathrm{d}}\right\rangle \\
& \times \exp \left[i \left(\varphi_{\Omega_{\mathrm{f}} \varepsilon_{\mathrm{f}} J_{\mathrm{f}}}-\varphi_{\left.\left.\Omega_{\mathrm{d} \varepsilon_{\mathrm{d}} J_{\mathrm{d}}}\right)\right]\left\langle\Omega_{\mathrm{d}}\left|(D)_{n}^{1}\right| \Omega_{\mathrm{f}}\right\rangle}\right.\right. \\
& \times\left(2 J_{\mathrm{f}}+1\right)^{1 / 2}\left(2 J_{\mathrm{d}}+1\right)(-1)^{2 J_{\mathrm{d}}}\left\{\begin{array}{cccc}
1 & 1 & K \\
J_{\mathrm{f}} & J_{\mathrm{i}} & J_{\mathrm{d}}
\end{array}\right\}\left(\begin{array}{cccc}
J_{\mathrm{i}} & 1 & J_{\mathrm{d}} \\
-\Omega_{\mathrm{i}} & m & \Omega_{\mathrm{d}}
\end{array}\right) \\
& \times\left.(-1)^{J_{\mathrm{d}}-\Omega_{\mathrm{d}}}\left(\begin{array}{cccc}
J_{\mathrm{d}} & 1 & J_{\mathrm{f}} \\
-\Omega_{\mathrm{d}} & m & \Omega_{\mathrm{f}}
\end{array}\right)\right|^{2} m=\Omega_{\mathrm{i}}-\Omega_{\mathrm{d}} ; \quad n=\Omega_{\mathrm{d}}-\Omega_{\mathrm{f}}
\end{aligned}
$$


Table I. - Explicit values of $\mathcal{F}_{k}$ defined by equation 14 , for various experimental arrangements. $\mathrm{e}_{\mathrm{i}}$ is the polarization of the incident photon and $\mathbf{e}_{\mathrm{f}}$ the polarization of the emitted photon. $(\mathbf{e}=\mathbf{X}$ or $\mathbf{Z}$ represents a linear polarization, while $\mathbf{e}=( \pm \mathbf{X}+i \mathbf{Y}) / \sqrt{2}$ represents a circular polarization.)

\begin{tabular}{|c|c|c|c|c|c|}
\hline EXPERIMENT & $\overrightarrow{\mathrm{e}}_{\mathrm{i}}$ & $\overrightarrow{\mathrm{e}}_{\mathrm{f}}$ & $\mathscr{F}_{0}$ & $F_{1}$ & $F_{2}$ \\
\hline$\stackrel{\stackrel{f e}{e}}{\longrightarrow}$ & $\vec{z}$ & $\vec{z}$ & $\frac{1}{3}$ & 0 & $\frac{2}{3}$ \\
\hline${\overrightarrow{e_{f}}}_{\vec{\gamma}}$ & $\overrightarrow{\mathbf{z}}$ & $\vec{x}$ & 0 & $\frac{1}{2}$ & $\frac{1}{2}$ \\
\hline & $\frac{\vec{x}+i \vec{y}}{\sqrt{2}}$ & $\vec{z}$ & 0 & $\frac{1}{2}$ & $\frac{1}{2}$ \\
\hline & $\frac{\vec{X}+i \vec{Y}}{\sqrt{2}}$ & $\vec{x}$ & $\frac{1}{6}$ & $\frac{1}{4}$ & $\frac{7}{12}$ \\
\hline & $\frac{\vec{X}+\vec{Y} \bar{Y}}{\sqrt{2}}$ & $\frac{\vec{X}+i \vec{\gamma}}{\sqrt{2}}$ & $\frac{1}{3}$ & $\frac{1}{2}$ & $\frac{1}{6}$ \\
\hline & $\frac{\vec{X}_{+i} \vec{Y}}{\sqrt{2}}$ & $\frac{-\vec{X}+i Y}{\sqrt{2}}$ & 0 & 0 & 1 \\
\hline
\end{tabular}

where $\varepsilon_{\mathrm{d}}$ and $\varepsilon_{\mathrm{f}}$ are the energies on the energy shell, i.e. $\varepsilon_{\mathrm{d}}=E_{\mathrm{i}}+\hbar c k_{\mathrm{i}}$ and $\varepsilon_{\mathrm{f}}=\varepsilon_{\mathrm{d}}-\hbar c k_{\mathrm{f}}$. In equation 16 $\varphi_{\Omega_{\mathrm{r} \varepsilon \mathrm{f} J} J_{\mathrm{f}}}$ and $\varphi_{\Omega_{\mathrm{d} \varepsilon_{\mathrm{d}} J_{\mathrm{d}}}}$ are the phases of the continuum wavefunctions for the intermediate and the final electronic states, respectively.

Equation 16 constitutes a quite complete and general result for photodissociation and fragments fluorescence. It was obtained under the following two assumptions :

i) the system is initially randomly oriented (sum over $M_{\mathrm{i}}$ ),

ii) the dissociation time is short compared with the fluorescence lifetime (Franck-Condon approximation for the fluorescence).

We shall delay the application of (16) to a particular molecular system, namely the case of $\mathrm{Ca}_{2}$, and consider now a limiting situation : the case in which dissociation proceeds fast as compared with the rotational motion of the molecule (Axial Recoil).

2.2 AXIAL RECOIL LIMIT. - In this limit, the transition matrix elements $\left\langle\left|(D)_{m}^{1}\right|\right\rangle$ and also $\varphi_{\Omega_{\mathrm{d}} \varepsilon_{\mathrm{d}} J_{\mathrm{d}}}$ can be considered to be independent of $J_{\mathrm{d}}$. In that case the sums over $J_{\mathrm{d}}$ and $J_{\mathrm{f}}$ in (16) can be carried out (cf. Appendix B) with the result :

$\frac{\mathrm{d} \sigma}{\mathrm{d} \hat{\omega}_{\mathrm{f}}}=2 \pi^{2} k_{\mathrm{i}} k_{\mathrm{f}}^{3} \Gamma_{\mathrm{d}}^{-1} \sum_{K} \mathcal{F}_{K}\left|\sum_{\Omega_{\mathrm{d}}}\left\langle\Omega_{\mathrm{i}} v_{\mathrm{i}} J_{\mathrm{i}}\left|(D)_{m}^{1}\right| \Omega_{\mathrm{d}} \varepsilon_{\mathrm{d}} J_{\mathrm{d}}\right\rangle\left\langle\Omega_{\mathrm{d}}\left|(D)_{n}^{1}\right| \Omega_{\mathrm{f}}\right\rangle\left(\begin{array}{ccc}1 & 1 & K \\ n & m & -(n+m)\end{array}\right)\right|^{2}$

with $m=\Omega_{\mathrm{i}}-\Omega_{\mathrm{d}}, n=\Omega_{\mathrm{d}}-\Omega_{\mathrm{f}}, J_{\mathrm{d}}=J_{\mathrm{i}}, \varepsilon_{\mathrm{d}}=E_{\Omega_{\mathrm{i}} v_{\mathrm{i}} J_{\mathrm{i}}}+\hbar c k_{\mathrm{i}}$.

Invoking now the Frančk-Condon approximation for the matrix element $\left\langle\left|(D)_{m}^{1}\right|\right\rangle=\left\langle\chi_{v_{\mathrm{i}} J_{\mathrm{i}}\left|\Omega_{\mathrm{f}}\right|} \mid \chi_{\varepsilon_{\mathrm{d}} J_{\mathrm{d}}\left|\Omega_{\mathrm{d}}\right|}\right\rangle$ $\left\langle\Omega_{\mathrm{i}}\left|(D)_{m}^{1}\right| \Omega_{\mathrm{d}}\right\rangle$ we can write :

$$
\left.\left.\frac{\mathrm{d} \sigma}{\mathrm{d} \hat{\omega}_{\mathrm{f}}} \propto \sum_{K} \mathscr{F}_{K}\right|_{\Omega_{\mathrm{d}}= \pm\left|\Omega_{\mathrm{d}}\right|}\left\langle\Omega_{\mathrm{i}}\left|(D)_{m}^{1}\right| \Omega_{\mathrm{d}}\right\rangle\left\langle\Omega_{\mathrm{d}}\left|(D)_{n}^{1}\right| \Omega_{\mathrm{f}}\right\rangle\left(\begin{array}{ccc}
1 & 1 & K \\
n & m & -(n+m)
\end{array}\right)\right|^{2}
$$

with $m=\Omega_{\mathrm{i}}-\Omega_{\mathrm{d}}$ and $n=\Omega_{\mathrm{d}}-\Omega_{\mathrm{f}}$. All the dependence on the final photon polarization $\mathrm{e}_{\mathrm{f}}$ is contained in the $\mathcal{F}_{K}$ factors (see Eq. 14 and table I). Therefore, from equation 18 it is easy to show that the polarization ratio $P=\left(I_{\|}-I_{\perp}\right) /\left(I_{\|}+I_{\perp}\right)$ will be independent of $J_{\mathrm{i}}$ in the axial recoil limit. Incidentally, equation 18 is the expression used by Vigue et al. [10] to interpret the results in $\mathrm{Ca}_{2}$ and in which they have neglected altogether the effect of rotation of the molecule.

Another important point to notice in equation 18 is the interference contribution to the cross section coming from the two $\Omega_{\mathrm{d}}= \pm\left|\Omega_{\mathrm{d}}\right|$ components. This interference was not taken into account by van Brunt and Zare [4]. Consider for example the case of a ${ }^{1} \Sigma \rightarrow^{1} \Pi \rightarrow{ }^{1} \Sigma$ transition. With the usual assumptions about electronic matrix elements [13] we get, from equation 18,

$$
\frac{\mathrm{d} \sigma}{\mathrm{d} \hat{\omega}_{\mathrm{f}}} \propto \frac{\mathscr{F}_{0}}{3}+\frac{\mathcal{F}_{2}}{30} .
$$

If instead we write equation 18 without the interference terms, i.e.

$$
\frac{\mathrm{d} \sigma}{\mathrm{d} \hat{\omega}_{\mathrm{f}}} \propto \sum_{K, \Omega_{\mathrm{d}}} \mathcal{F}_{K}\left|\left\langle\Omega_{\mathrm{i}}\left|(D)_{m}^{1}\right| \Omega_{\mathrm{d}}\right\rangle\left\langle\Omega_{\mathrm{d}}\left|(D)_{n}^{1}\right| \Omega_{\mathrm{f}}\right\rangle\left(\begin{array}{ccc}
1 & 1 & K \\
n & m & -(n+m)
\end{array}\right)\right|^{2}
$$


we get for the same case

$$
\frac{\mathrm{d} \sigma}{\mathrm{d} \hat{\omega}_{\mathrm{f}}} \propto \frac{\mathcal{F}_{0}}{3}+\frac{\mathcal{F}_{1}}{6}+\frac{\mathcal{F}_{2}}{30} .
$$

The results, equations 19 and 21 , corresponding to different experimental conditions are presented in table II. We note that large discrepancies between the results obtained with and without interference exist.

Table II. - The polarization ratio

$$
P=\left(I_{\|}-I_{\perp}\right) /\left(I_{\|}+I_{\perp}\right)
$$

corresponding to different experiments is given in the limiting case of the axial recoil for $a^{1} \Sigma \rightarrow{ }^{1} \Pi \rightarrow{ }^{1} \Sigma$ transition. The polarization vectors are defined like in table I. Values of $P$ are calculated with and without

\begin{tabular}{|c|c|c|c|c|}
\hline \multicolumn{3}{|c|}{ GEOMETRY } & \multicolumn{2}{|c|}{ POLARIZATION RATE } \\
\hline \multirow[t]{2}{*}{$\begin{array}{c}\text { EXCITATION } \\
\text { POLARTZATION } \\
\vec{e}_{i}\end{array}$} & \multicolumn{2}{|c|}{$\begin{array}{c}\text { DETECTION } \\
\text { POLARTZATION } \\
\overrightarrow{\mathbf{e}}_{\mathbf{f}}\end{array}$} & \multirow[t]{2}{*}{$\begin{array}{c}\text { WITH } \\
\text { INTERFERENCE }\end{array}$} & \multirow[t]{2}{*}{$\begin{array}{l}\text { WITHOUT } \\
\text { INTERFERENCE }\end{array}$} \\
\hline & $I_{1}$ & $I_{1}$ & & \\
\hline$\vec{r}$ & $\vec{z}$ & $\overrightarrow{\mathrm{x}}$ & $\frac{7}{9} \simeq 0.78$ & $\frac{1}{7} \simeq 0.14$ \\
\hline$\frac{\vec{x}+1 \vec{v}}{\sqrt{2}}$ & $\vec{x}$ & $\overrightarrow{\mathbf{z}}$ & $\frac{7}{11} \approx 0.64$ & $\frac{1}{13} \approx 0.08$ \\
\hline$\frac{\vec{x}+i \vec{y}}{\sqrt{2}}$ & $\frac{\overrightarrow{\mathrm{x}}+1 \overrightarrow{\mathrm{y}}}{\sqrt{2}}$ & $\frac{-\vec{x}+i \vec{Y}}{\sqrt{2}}$ & $\frac{5}{9} \approx 0.56$ & $\frac{5}{7} \simeq 0.71$ \\
\hline
\end{tabular}
interference between the $\Omega_{\mathrm{d}}= \pm 1$ states.

In particular, for excitation with linearly polarized light, the result without interference is the one obtained by van Brunt and Zare [4], namely $P=14 \%$, while the result with interference is $P=78 \%$. We conclude that the interference between photodissociation amplitudes corresponding to different $\Omega_{\mathrm{d}}= \pm\left|\Omega_{\mathrm{d}}\right|$ components of the electronic angular momentum is crucial for the correct calculation of polarization ratios.
It should be noticed however, that those coherence effects will not be noticeable in the polarization of the light emitted if $\Omega_{\mathrm{d}}>1$.

\section{Photofragment fluorescence in the case of predisso- ciation.}

In the case of photopredissociation, we shall describe the molecular plus field Hamiltonian $H_{0}=H_{\mathrm{M}}+H_{\mathrm{R}}$ by four sets of states :a) $\left|\alpha_{\mathrm{i}} ; \mathbf{k}_{\mathrm{i}} \mathbf{e}_{\mathbf{i}}\right\rangle$ describing the initial bound state plus a photon of energy $\hbar c k_{\mathrm{i}}$ and polarization $\left.e_{i} ; b\right)$ an intermediate $\left|\alpha_{s} ; 0\right\rangle$ bound electronic state which can be excited from the initial states by photon absorption; $c$ ) a dissociative state $\left|\alpha_{d} ; 0\right\rangle$ which predissociates $\left|\alpha_{s}\right\rangle$ and produces excited fragments $; d$ ) a final $\left|\alpha_{\mathrm{f}} ; \mathbf{k}_{\mathrm{f}} \mathbf{e}_{\mathrm{f}}\right\rangle$ state obtained by emission of a photon from $\left|\alpha_{d} ; 0\right\rangle$. We assume in this section that the dissociative states $\left|\alpha_{d} ; 0\right\rangle$ cannot be excited directly from the initial states, i.e. we exclude from our treatment the case of interference between direct dissociation and predissociation which leads to Fano's lineshapes. From equations 4 and 5 , we have

$$
\begin{aligned}
\frac{\mathrm{d} \sigma}{\mathrm{d} \hat{\omega}_{\mathrm{f}}}=\frac{2 \pi k_{\mathrm{f}}^{2}}{(\hbar c)^{2}} \mid\left\langle\alpha_{\mathrm{i}} ; \mathbf{k}_{\mathrm{i}} \mathbf{e}_{\mathrm{i}}\right| H_{\text {int. }} & G^{+}(E) H_{\text {int. }} \\
& \times\left.\left|\alpha_{\mathrm{f}} ; \mathbf{k}_{\mathrm{f}} \mathbf{e}_{\mathrm{f}}\right\rangle\right|^{2} .
\end{aligned}
$$

The total Hamiltonian is now $H=H_{0}+H_{\text {int. }}+H_{\mathrm{v}}$ where $H_{\mathrm{v}}$ is the intramolecular coupling responsible for predissociation. Defining the projection operators

$$
\begin{aligned}
& P=\sum_{\alpha_{\mathrm{s}}}\left|\alpha_{\mathrm{s}} ; 0\right\rangle\left\langle 0 ; \alpha_{\mathrm{s}}\right| \\
& Q=1-P
\end{aligned}
$$

and using the relation [18]

$$
\begin{aligned}
P G Q= & P\left(E^{+}-P H_{0} P-P R P\right)^{-1} \times \\
& \times P H_{\mathrm{v}} Q\left(E^{+}-Q H Q\right)^{-1} Q
\end{aligned}
$$

where $R$ is the level shift operator

$$
R=H_{\mathrm{v}}+H_{\text {int. }}+\left(H_{\mathrm{v}}+H_{\text {int. }}\right)\left(E^{+}-Q H Q\right)^{-1}\left(H_{\mathrm{v}}+H_{\text {int }}\right)
$$

it is obtained,

$$
\begin{aligned}
\frac{\mathrm{d} \sigma}{\mathrm{d} \hat{\omega}_{\mathrm{f}}}=\frac{2 \pi k_{\mathrm{f}}^{2}}{(\hbar c)^{2}} \mid \sum_{\alpha_{\mathrm{s}} \alpha_{\mathrm{d}}}\left\langle\alpha_{\mathrm{i}} ; \mathbf{k}_{\mathrm{i}} \mathbf{e}_{\mathrm{i}}\left|H_{\text {int. }}\right| \alpha_{\mathrm{s}} ; 0\right. & >\left(E-E_{\mathrm{s}}-\Delta_{\mathrm{s}}+i \Gamma_{\mathrm{s}}\right)^{-1} \times \\
& \times\left.\left\langle\alpha_{\mathrm{s}}\left|H_{\mathrm{v}}\right| \alpha_{\mathrm{d}}\right\rangle\left(E^{+}-E_{\mathrm{d}}+i \Gamma_{\mathrm{d}}\right)^{-1}\left\langle\alpha_{\mathrm{d}} ; 0\left|H_{\text {int. }}\right| \alpha_{\mathrm{f}} ; \mathbf{k}_{\mathrm{f}} \mathbf{e}_{\mathrm{f}}\right\rangle\right|^{2}
\end{aligned}
$$

where $\Delta_{\mathrm{s}}$ and $\Gamma_{\mathrm{s}}$ are the real and imaginary parts of PRP, respectively.

We now specify the wavefunctions as in equation 11 . Since $H_{\mathrm{v}}$ is an intramolecular coupling, we have

$$
\left\langle\Omega_{\mathrm{s}} v_{\mathrm{s}} J_{\mathrm{s}} M_{\mathrm{s}}\left|H_{\mathrm{v}}\right| \Omega_{\mathrm{d}} \varepsilon_{\mathrm{d}} J_{\mathrm{d}} M_{\mathrm{d}}\right\rangle=\left\langle\Omega_{\mathrm{s}} v_{\mathrm{s}} J_{\mathrm{s}}\left|H_{\mathrm{v}}\right| \Omega_{\mathrm{d}} \varepsilon_{\mathrm{d}} J_{\mathrm{d}}\right\rangle \delta_{J_{\mathrm{s}} J_{\mathrm{d}}} \delta_{M_{\mathrm{s}} M_{\mathrm{d}}} .
$$


Using this result together with equations 8,11 and performing the sum over $M_{\mathrm{i}}, M_{\mathrm{d}}$ and $M_{\mathrm{f}}$, it is obtained

$$
\begin{aligned}
& \frac{\mathrm{d} \sigma}{\mathrm{d} \hat{\omega}_{\mathrm{f}}}=2 \pi k_{\mathrm{i}} \int \mathrm{d} \varepsilon_{\mathrm{f}} \sum_{J_{\mathrm{f}}} \sum_{\Omega} k_{\mathrm{f}}^{3} \mathcal{F}_{K} \mid \sum_{\Omega_{\mathrm{s}} v_{\mathrm{s}} J_{\mathrm{s}}} \sum_{\Omega_{\mathrm{d}}} \int \mathrm{d} \varepsilon_{\mathrm{d}} \\
& \left\langle\Omega_{\mathrm{i}} v_{\mathrm{i}} J_{\mathrm{i}}\left|(D)_{m}^{1}\right| \Omega_{\mathrm{s}} v_{\mathrm{s}} J_{\mathrm{s}}\right\rangle\left\langle\Omega_{\mathrm{s}} v_{\mathrm{s}} J_{\mathrm{s}}\left|H_{\mathrm{v}}\right| \Omega_{\mathrm{d}} \varepsilon_{\mathrm{d}} J_{\mathrm{s}}\right\rangle \\
& \left\langle\Omega_{\mathrm{d}} \varepsilon_{\mathrm{d}} J_{\mathrm{s}}\left|(D)_{n}^{1}\right| \Omega_{\mathrm{f}} \varepsilon_{\mathrm{f}} J_{\mathrm{f}}\right\rangle\left(E-E_{\Omega_{\mathrm{s}} v_{\mathrm{s}} J_{\mathrm{s}}}-\Delta_{\Omega_{\mathrm{s}} v_{\mathrm{s}} J_{\mathrm{s}}}+i \Gamma_{\Omega_{\mathrm{s}} v_{\mathrm{s}} J_{\mathrm{s}}}\right)^{-1} \\
& \left(E-\varepsilon_{\mathrm{d}}+i \Gamma_{\mathrm{d}}\right)^{-1}\left(2 J_{\mathrm{i}}+1\right)^{1 / 2}\left(2 J_{\mathrm{f}}+1\right)^{1 / 2}(-1)^{2 J_{\mathrm{s}}}\left(2 J_{\mathrm{s}}+1\right)\left\{\begin{array}{ccc}
1 & 1 & K \\
J_{\mathrm{f}} & J_{\mathrm{i}} & J_{\mathrm{s}}
\end{array}\right\} \\
& \left.\left(\begin{array}{ccc}
J_{\mathrm{i}} & 1 & J_{\mathrm{s}} \\
-\Omega_{\mathrm{i}} & m & \Omega_{\mathrm{s}}
\end{array}\right)(-1)^{J_{\mathrm{s}}-\Omega_{\mathrm{d}}}\left(\begin{array}{ccc}
J_{\mathrm{s}} & 1 & J_{\mathrm{f}} \\
-\Omega_{\mathrm{d}} & n & \Omega_{\mathrm{f}}
\end{array}\right)\right|^{2} \\
& m=\Omega_{\mathrm{i}}-\Omega_{\mathrm{s}} ; \quad n=\Omega_{\mathrm{d}}-\Omega_{\mathrm{f}} .
\end{aligned}
$$

This constitutes the general result. We now consider two limiting cases : i) fast; ii) slow predissociation.

i) Fast predissociation. - We define fast predissociation when the predissociation is fast as compared with the rotational motion. We shall then have the predissociation rate much larger than the rotational spacings in the region of interest, i.e.

$$
\Gamma_{\Omega_{\mathbf{s}} v_{\mathbf{s}} J_{\mathbf{s}}} \gg E_{\Omega_{\mathbf{s}} v_{\mathbf{s}} J_{\mathbf{s}}}-E_{\Omega_{\mathbf{s}} v_{\mathbf{s}} J_{\mathbf{s}}^{\prime}} ; \quad J_{\mathrm{s}}^{\prime}=J_{\mathrm{s}} \pm 1
$$

We may then replace the energy denominator $\left(E-E_{\Omega_{\mathbf{s}} v_{\mathbf{s}} J_{\mathbf{s}}}-\Delta_{\Omega_{\mathbf{s}} v_{\mathbf{s}} J_{\mathbf{s}}}+i \Gamma_{\Omega_{\mathbf{s}} v_{\mathbf{s}} J_{\mathbf{s}}}\right)$ by $\Gamma_{\Omega_{\mathbf{s}} v_{\mathbf{s}} J_{\mathbf{s}}}$. Making use again of the axial recoil approximation and Franck-Condon, we get

$$
\frac{\mathrm{d} \sigma}{\mathrm{d} \hat{\omega}_{\mathrm{f}}} \propto \sum_{K} \mathcal{F}_{K}\left|\sum_{\Omega_{\mathrm{s}}} \sum_{\Omega_{\mathrm{d}}}\left\langle\Omega_{\mathrm{i}}\left|(D)_{m}^{1}\right| \Omega_{\mathrm{s}}\right\rangle\left\langle\Omega_{\mathrm{s}}\left|H_{\mathrm{v}}\right| \Omega_{\mathrm{d}}\right\rangle\left\langle\Omega_{\mathrm{d}}\left|(D)_{n}^{1}\right| \Omega_{\mathrm{f}}\right\rangle\left(\begin{array}{ccc}
1 & 1 & K \\
n & m & -(n+m)_{\mathrm{s}}
\end{array}\right)\right|^{2} .
$$

We notice from this result that in the case of homogeneous predissociation $\left(\Omega_{\mathrm{s}}=\Omega_{\mathrm{d}}\right)$ we have the same result as in the case of direct photodissociation.

ii) Slow predissociation. - This is the opposite limit and it corresponds to

$$
\Gamma_{\Omega_{\mathrm{s}} v_{\mathrm{s}} J_{\mathrm{s}}} \ll E_{\Omega_{\mathrm{s}} v_{\mathrm{s}} J_{\mathrm{s}}}-E_{\Omega_{\mathrm{s}} v_{\mathbf{s}} J_{\mathrm{s}}^{\prime}} ; \quad J_{\mathrm{s}}^{\prime}=J_{\mathrm{s}} \pm 1
$$

In this limit it is possible to select a particular ro-vibrational state in excitation. The atomic fragments polarization is then given by formula 29 , without the summation on $J_{\mathrm{s}}$. The interference effect discussed in the case of photodissociation appears also in formula 29 in the summations on $\Omega_{\mathrm{s}}$ and $\Omega_{\mathrm{d}}$. However the influence of the summation is rather trivial. If we consider the case corresponding to $\Omega_{\mathrm{i}}=\Omega_{\mathrm{f}}=0$ and $\left|\Omega_{\mathrm{d}}\right|=\left|\Omega_{\mathrm{s}}\right|=1$, the two interference terms $+\left|\Omega_{\mathrm{d}}\right|$ and $-\left|\Omega_{\mathrm{d}}\right|$ are or equal, or of opposite sign, and they only ensure that $J_{\mathrm{f}}$ has the same parity as $J_{\mathrm{i}}$. Indeed, for the following transitions $:{ }^{1} \Sigma^{+} \stackrel{\text { excitation }}{\longrightarrow}{ }^{1} \Pi \stackrel{\text { predissociation }}{\longrightarrow}{ }^{1} \Pi \stackrel{\text { fluorescence }}{\longrightarrow}{ }^{1} \Sigma^{+}$ only $\mathbf{R}$ or $\mathbf{P}$ lines (respectively $\mathrm{Q}$ lines) are observed in the fluorescence, for an excitation by $\mathbf{R}$ or $\mathbf{P}$ lines (respectively $\mathrm{Q}$ lines).

Therefore in (29), it is possible to forget the summations on $\pm\left|\Omega_{\mathrm{d}}\right|$ and $\pm\left|\Omega_{\mathrm{s}}\right|$ considering only the $J_{\mathrm{f}}$ values corresponding to non vanishing transitions. Remarking that for predissociation in absence of external fields, $J_{\mathrm{s}}=J_{\mathrm{d}}$, we finally obtain

$$
\begin{aligned}
& \frac{\mathrm{d} \sigma}{\mathrm{d} \hat{\omega}_{\mathrm{f}}} \propto \sum_{J_{\mathrm{f}}} \sum_{K} \mathcal{F}_{K}\left|\left\{\begin{array}{ccc}
1 & 1 & K \\
J_{\mathrm{f}} & J_{\mathrm{i}} & J_{\mathrm{s}}
\end{array}\right\}\right|^{2} \times \\
& \times\left(2 J_{\mathrm{s}}+1\right)\left(2 J_{\mathrm{i}}+1\right)\left(\begin{array}{ccc}
J_{\mathrm{i}} & 1 & J_{\mathrm{s}} \\
-\left|\Omega_{\mathrm{i}}\right| & m & \left|\Omega_{\mathrm{s}}\right|
\end{array}\right)^{2}\left(2 J_{\mathrm{s}}+1\right)\left(2 J_{\mathrm{f}}+1\right)\left(\begin{array}{ccc}
J_{\mathrm{s}} & 1 & J_{\mathrm{f}} \\
-\left|\Omega_{\mathrm{d}}\right| & n & \left|\Omega_{\mathrm{f}}\right|
\end{array}\right)^{2} \\
& m=\left|\Omega_{\mathrm{i}}\right|-\left|\Omega_{\mathrm{s}}\right| \text { and } n=\left|\Omega_{\mathrm{d}}\right|-\left|\Omega_{\mathrm{f}}\right| .
\end{aligned}
$$

The first part of equation 33 represents the polarization of each transition $J_{i} \rightarrow J_{s} \rightarrow J_{f}$ and the second one their intensities (Hönl London factors).

In fact, formula 33 gives also the polarization of the fluorescence light in absence of predissociation. Howe-

JOURNAL DE PHYSIQUE. - T. 44, No 11, NOVEMBRE 1983 ver in this last case, it is possible to select a $\mathbf{P}, \mathbf{Q}$, or $\mathbf{R}$ line at the detection and the summation on $J_{\mathrm{f}}$ is 
usually omitted. The polarization rates corresponding to various transitions have been calculated and tabulated by various authors $[14,15]$.

In the case of photopredissociation, the selectivity at the detection is impossible and the summation on $J_{\mathrm{f}}$ must be performed. Since this summation is incoherent the polarization of the atomic fragments can easily be obtained by averaging the intensities of the various transitions weighted by the Hönl London factors.

As an illustration table III gives the polarization rates of the atomic fragments in the limit of high $J$ values for two types of transitions in the case of homogeneous predissociation. In transition $(a)$, for a $\mathrm{Q}$ exciting line $J_{\mathrm{f}}=J_{\mathrm{d}}$ and for a $\mathrm{P}$ or $\mathrm{R}$ exciting line $J_{\mathrm{f}}=J_{\mathrm{d}} \pm 1$. In transition $(b)$, we have always $J_{\mathrm{f}}=$ $J_{\mathrm{d}} \pm 1$ (for $J_{\mathrm{d}} \rightarrow \infty$ ).

Finally it must be remarked that for excitation with circularly polarized' light, the polarization

Table III. - Polarization ratios of atomic fragments in a photopredissociation for two types of transitions. The exciting light is linearly polarized along $\mathrm{O} z$ and $P=\left(I_{\|}-I_{\perp}\right) /\left(I_{\|}+I_{\perp}\right)$. The polarization ratios are given in the limit $J \rightarrow \infty$.

\begin{tabular}{|c|c|c|}
\hline $\begin{array}{c}\text { Excitation } \\
\text { line }\end{array}$ & 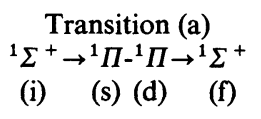 & $\begin{array}{c}\text { Transition (b) } \\
{ }^{1} \Sigma^{+} \rightarrow^{1} \Pi--^{1} \Pi \rightarrow{ }^{1} \Pi \\
\text { (i) } \\
\text { (s) (d) }\end{array}$ \\
\hline $\begin{array}{l}\mathrm{Q} \text { branch } \\
\mathrm{P} \text { or } \mathrm{R} \text { branch }\end{array}$ & $\begin{array}{l}P=\frac{1}{2} \\
P=\frac{1}{7}\end{array}$ & $\begin{array}{l}P=-\frac{1}{3} \\
P=\frac{1}{7}\end{array}$ \\
\hline
\end{tabular}

$P=\left(I_{\sigma^{+}}-I_{\sigma^{-}}\right) /\left(I_{\sigma^{+}}+I_{\sigma^{-}}\right)$detected in the direction of exciting beam is always equal to zero in the limit of high $J$ values. This is due to the fact that the sign of this polarization rate is opposite for $\mathbf{P}$ and $\mathbf{R}$ lines and varies as $1 / J_{d}$ or $1 / J_{d}^{2}$ for $Q$ lines [16].

\section{Relationship between photofragments polarization and angular distributions.}

4.1 AXIAL ReCOIL APPROXIMATION. - In sections 2 and 3 we have presented the complete quantum mechanical treatment of the polarization of the photofragments fluorescence. It seems now interesting to relate this treatment to the original approach of van Brunt and Zare [4]. They utilized the following method : i) the atomic fluorescence polarization is calculated for each possible orientation of the molecule; ii) this polarization is averaged over the angular distribution of the fragments $f_{\Omega_{\mathrm{i}} \Omega_{\mathrm{d}}}(\theta, \varphi)$. This relationship is easy to derive in the case of direct photodissociation and axial recoil situations. The detailed calculation is given in appendix $\mathrm{E}$. The final result in the case of $\Omega_{\mathrm{i}}=\Omega_{\mathrm{f}}=0$ and $\left|\Omega_{\mathrm{d}}\right|=1$ is :

$\frac{\mathrm{d} \sigma}{\mathrm{d} \hat{\omega}_{\mathrm{f}}}=\int \mathrm{d} \hat{\omega}\left|\sum_{\Omega_{\mathrm{d}}= \pm 1} g_{\Omega_{\mathrm{i}} \Omega_{\mathrm{d}}}(\theta, \varphi) a_{\Omega_{\mathrm{d}} \Omega_{\mathrm{f}}}(\theta, \varphi)\right|^{2}$

where $\mathrm{d} \hat{\omega}=\sin \theta \mathrm{d} \theta \mathrm{d} \varphi$ is the solid angle element, and $\theta, \varphi$ the polar angles specifying the orientation of the internuclear axis with respect to the laboratory frame $\mathrm{O} X Y Z$. The functions $g_{\Omega_{\mathrm{i}} \Omega_{\mathrm{d}}}(\theta, \varphi)$ and $a_{\Omega_{\mathrm{d}} \Omega_{\mathrm{f}}}(\theta, \varphi)$ are given by

$$
\begin{aligned}
& a_{\Omega_{\mathrm{d}} \Omega_{\mathrm{f}}}(\theta, \varphi)=\sum_{q}(-1)^{q}\left(e_{\mathrm{f}}^{*}\right)_{q}^{1} D_{-q n}(\varphi, \theta, 0)\left\langle\Omega_{\mathrm{d}}\left|(D)_{n}^{1}\right| \Omega_{\mathrm{f}}\right\rangle \\
& g_{\Omega_{\mathrm{i}} \Omega_{\mathrm{d}}}(\theta, \varphi)=\sum_{p}(-1)^{p}\left(e_{\mathrm{i}}\right)_{p}^{1} D_{-p m}^{1}(\varphi, \theta, 0)\left\langle\Omega_{\mathrm{i}}\left|(D)_{m}^{1}\right| \Omega_{\mathrm{d}}\right\rangle \quad n=\Omega_{\mathrm{d}}-\Omega_{\mathrm{f}} ; \quad m=\Omega_{\mathrm{i}}-\Omega_{\mathrm{d}} .
\end{aligned}
$$

It should be noticed at this point, that the angular distribution of the photofragments $f_{\Omega_{\mathrm{i}} \Omega_{\mathrm{d}}}(\theta, \varphi)$ is related to $g_{\Omega_{\mathrm{i}} \Omega_{\mathrm{d}}}(\theta, \varphi)$ in the axial recoil limit, by :

$$
f_{\Omega_{\mathrm{i}} \Omega_{\mathrm{d}}}(\theta, \varphi)=\left|g_{\Omega_{\mathrm{i}} \Omega_{\mathrm{d}}}(\theta, \varphi)\right|^{2}
$$

The $g_{\Omega_{\mathrm{i}} \Omega_{\mathrm{d}}}(\theta, \varphi)$ function appears as an amplitude of angular distribution. The interference effect between $+\Omega_{\mathrm{d}}$ and $-\Omega_{\mathrm{d}}$ emerges clearly from equation 34 which is completely equivalent to equation 18 . Equation 34 shows that in the presence of interference effect between $+\Omega_{\mathrm{d}}$ and $-\Omega_{\mathrm{d}}$ it is not possible to relate the atomic photofragment polarization to the angular distribution $f_{\Omega_{\mathrm{i}} \Omega_{\mathrm{d}}}(\theta, \varphi)$ in the way that van Brunt and Zare did [4]. On the other hand when no interference exists this relationship is possible. Consider for example the case $\Omega_{\mathrm{i}}=$ $\Omega_{\mathrm{f}}=\Omega_{\mathrm{d}}=0$ or $\Omega_{\mathrm{i}}=0$ and $\left|\Omega_{\mathrm{d}}\right|=\left|\Omega_{\mathrm{f}}\right|=1$. Equation 34 then reduces to

$$
\frac{\mathrm{d} \sigma}{\mathrm{d} \hat{\omega}_{\mathrm{f}}} \propto \int \mathrm{d} \omega f_{\Omega_{\mathrm{i}} \Omega_{\mathrm{d}}}(\theta, \varphi)\left|a_{\Omega_{\mathrm{d}} \Omega_{\mathrm{f}}}(\theta, \varphi)\right|^{2}
$$

which is exactly the relationship used by van Brunt and Zare [4] : $a_{\Omega_{\mathrm{d}} \Omega_{\mathrm{f}}}$ is the projection of the induced dipole in the molecular frame on the direction of the detected polarization $\mathbf{e}_{\mathrm{f}}, \mathcal{D}_{-q n}^{1}(\varphi, \theta, 0)$ being the rotation matrix between the laboratory and the molecular frames. 
In the particular case of linearly polarized exciting light $\left(\mathrm{e}_{\mathrm{i}} / / \mathrm{OZ}\right)$, and for $\Omega_{\mathrm{i}}=\Omega_{\mathrm{f}}=0$ and $\left|\Omega_{\mathrm{d}}\right|=1$. we have the following results :

$$
\begin{aligned}
g_{01}(\theta, \varphi) & =\frac{\sin \theta}{\sqrt{2}}\left\langle\Omega_{\mathrm{i}}=0\left|(D)_{-1}^{1}\right| \Omega_{\mathrm{d}}=1\right\rangle \\
g_{0-1}(\theta, \varphi) & =-\frac{\sin \theta}{\sqrt{2}}\left\langle\Omega_{\mathrm{i}}=0\left|(D)_{1}^{1}\right| \Omega_{\mathrm{d}}=-1\right\rangle .
\end{aligned}
$$

It is then possible to factorize in equation 34 , the angular distribution $f_{01}(\theta, \varphi)$ which in this case is proportional to $\sin ^{2} \theta$. Equation 34 may then be written :

$$
\frac{\mathrm{d} \sigma}{\mathrm{d} \hat{\omega}_{\mathrm{f}}} \propto \int \mathrm{d} \hat{\omega} f_{01}(\theta, \varphi)\left|a_{10}(\theta, \varphi)-a_{-10}(\theta, \varphi)\right|
$$

This is the basic equation used by Vigue et al. [10] in their treatment of $\mathrm{Ca}_{2}$ experiment. However this approach is not general and cannot be applied to most of the other geometrical arrangements. For example, if we consider a circularly polarized exciting beam propagating in the $\mathrm{OZ}$ direction, we get

$$
\begin{gathered}
g_{01}(\theta, \varphi)=-\mathrm{e}^{i \varphi}\left(\frac{1-\cos \theta}{2}\right)\left\langle\Omega_{\mathrm{i}}=0\left|(D)_{1}^{1}\right| \Omega_{\mathrm{d}}=-1\right\rangle \\
g_{0-1}(\theta, \varphi)=-\mathrm{e}^{i \varphi}\left(\frac{1+\cos \theta}{2}\right)\left\langle\Omega_{\mathrm{i}}=0\left|(D)_{-1}^{1}\right| \Omega_{\mathrm{d}}=1\right\rangle
\end{gathered}
$$

and in this case it is not possible to write the fluorescence cross section $\mathrm{d} \sigma / \partial \hat{\omega}_{\mathrm{f}}$ as an integral over the angular distribution $f_{01}(\theta, \varphi)$.

4.2 Polarization in the Case of a Rotating molecule. - For simplicity, we shall discuss here only the case of $\Omega_{\mathrm{i}}=\Omega_{\mathrm{f}}=0$ and $\left|\Omega_{\mathrm{d}}\right|=1\left({ }^{1} \Sigma \rightarrow^{1} \Pi \rightarrow{ }^{1} \Sigma\right.$ transitions). This is the case of $\mathrm{Ca}_{2}$ studied in reference 10. Using equation 16, we can express the fluorescence intensity for any polarization as a function of the phases $\varphi_{\Omega_{\mathrm{d} \varepsilon_{\mathrm{d}} J_{\mathrm{d}}}}$. To introduce the classical rotation angle $\alpha$, we use the relation C.4 of appendix C, which gives :

$$
\varphi_{\Omega_{\mathrm{d} \varepsilon_{\mathrm{d}} J_{\mathrm{d}}}}-\varphi_{\Omega_{\mathrm{d}} \varepsilon_{\mathrm{d}} J_{\mathrm{i}}}=\alpha\left(J_{\mathrm{d}}-J_{\mathrm{i}}\right) .
$$

(This linearization is accurate as long as $\alpha$ is not too large, i.e. $\alpha \lesssim 2 \pi$ ). The fluorescence intensity is then given by

$$
I \propto \sum_{K} \mathscr{F}_{K} \sum_{J_{\mathrm{f}}}\left|\sum_{\Omega_{\mathrm{d}}= \pm 1} S\left(J_{\mathrm{f}}, J_{\mathrm{i}}, \Omega_{\mathrm{d}}, K\right)\right|^{2}
$$

where

$$
\begin{aligned}
& S\left(J_{\mathrm{f}}, J_{\mathrm{i}}, \Omega_{\mathrm{d}}, K\right)=\sum_{J_{\mathrm{d}}}(-1)^{J_{\mathrm{d}}}\left(2 J_{\mathrm{d}}+1\right)\left(\begin{array}{ccc}
J_{\mathrm{i}} & 1 & J_{\mathrm{d}} \\
0 & -\Omega_{\mathrm{d}} & \Omega_{\mathrm{d}}
\end{array}\right) \times \\
& \times\left(\begin{array}{ccc}
J_{\mathrm{d}} & 1 & J_{\mathrm{f}} \\
-\Omega_{\mathrm{d}} & \Omega_{\mathrm{d}} & 0
\end{array}\right)\left\{\begin{array}{ccc}
1 & 1 & K \\
J_{\mathrm{f}} & J_{\mathrm{i}} & J_{\mathrm{d}}
\end{array}\right\} \mathrm{e}^{i \alpha\left(J_{\mathrm{i}}-J_{\mathrm{d}}\right)} .
\end{aligned}
$$

This can be calculated for any value of $J_{i}$ using the well-known formulae for 3-j and 6-j coefficients [17]. Using the fact that $J_{\mathrm{i}}$ is usually large, we may calculate the $J_{i} \rightarrow \infty$ limit using the limiting expressions deduced from reference 17 . The $S$ values obtained this way are given in table IV as a function of $\alpha$. From these results it is possible now to calculate the intensity $I$ using the $\mathcal{F}_{K}$ values from table $\mathrm{I}$. We may even drop the interference $\Omega_{\mathrm{d}}= \pm 1$ if we wish to reproduce the results of van Brunt and Zare [4].
In the case of linearly polarized light (first geometrical arrangement in table II) we get

$$
P=\left[1+3\left(\cos \alpha+\cos ^{2} \alpha\right)\right] /\left(7+\cos \alpha+\cos ^{2} \alpha\right)
$$

with the $\Omega_{\mathrm{d}}= \pm 1$ interference, and

$$
P=\left(3 \cos ^{2} \alpha-1\right) /\left(13+\cos ^{2} \alpha\right)
$$

without the interference. In the present case, the ani- 
Table IV.

$$
\begin{array}{lcc}
K & J_{\mathrm{f}}-J_{\mathrm{i}} & S\left(J_{\mathrm{f}}, J_{\mathrm{i}}, 1, K\right) \mid \times \sqrt{2 J_{\mathrm{i}}+1} \\
\hline 0 & - & - \\
1 & 0 & (1+\cos \alpha) /(2 \sqrt{3}) \\
1 & 0 & \sin \alpha /(2 \sqrt{6}) \\
2 & \pm 1 & \sqrt{1+\cos \alpha /(2 \sqrt{6})} \\
2 & 0 & \sqrt{1-\cos \alpha) /(2 \sqrt{30})} \\
2 & \pm 1 & 1 /(4 \sqrt{5}) \\
S\left(J_{\mathrm{f}}, J_{\mathrm{i}},-1, K\right)=(-1)^{J_{\mathrm{f}}-J_{\mathrm{i}}} S\left(J_{\mathrm{f}}, J_{\mathrm{i}}, 1, K\right) . \\
S\left(J_{\mathrm{f}}, J_{\mathrm{i}}, \Omega_{\mathrm{d}}, K\right) \text { as a function of } K,\left(J_{\mathrm{f}}-J_{\mathrm{i}}\right) \text { in the large } J_{\mathrm{i}}
\end{array}
$$
limit.

sotropy parameter of the fragment distribution $\beta$ is related to the classical rotation angle by $[18,19]$

$$
\beta=-P_{2}(\cos \alpha)=\left(1-3 \cos ^{2} \alpha\right) / 2 \text {. }
$$

The van Brunt and Zare's result for this case is [4]

$$
P=-\frac{3 \beta}{20-\beta}=\frac{3 \cos ^{2} \alpha-1}{13+\cos ^{2} \alpha}
$$

which is precisely the result obtained above without the interference between $\Omega_{\mathrm{d}}= \pm 1$ (see Eq. 47). This is a direct quantum mechanical confirmation of the relationship between $P$ and $\beta$, in the absence of interference and in the limit in which the rotation angle can be defined.

On the other hand, the calculation taking into account the interference effect produces a term linear in $\cos \alpha$, while $\beta$ depends only on $\cos ^{2} \alpha$. This demonstrates once more that the knowledge of $\beta$ is not sufficient to calculate $P$. In figure 1 we have represented $P$ and $\beta$ according to equations 46 and 48 as a function of $\alpha$. In what follows we shall present a classical interpretation of the $\alpha$ dependence of $P$.

\subsection{Classical interpretation OF THE DEPENDENCE} OF THE POLARIZATION RATIO ON THE ROTATION ANGLE. - Let us first discuss the physical meaning of the quantum mechanical calculations. The process studied has three steps :

i) excitation of a dipole perpendicular to the internuclear axis $\left({ }^{1} \Sigma \rightarrow{ }^{1} \Pi\right.$ transition);

ii) fast dissociation, and rotation around $\mathbf{J}$, the total angular momentum of the molecule. The replacement of the exact wavefunctions by their asymptotic expressions (cf. appendix C) means that dissociation and rotation are almost completely accomplished before emission starts;

iii) radiation of the dipole; the internuclear axis is fixed at its asymptotic position.

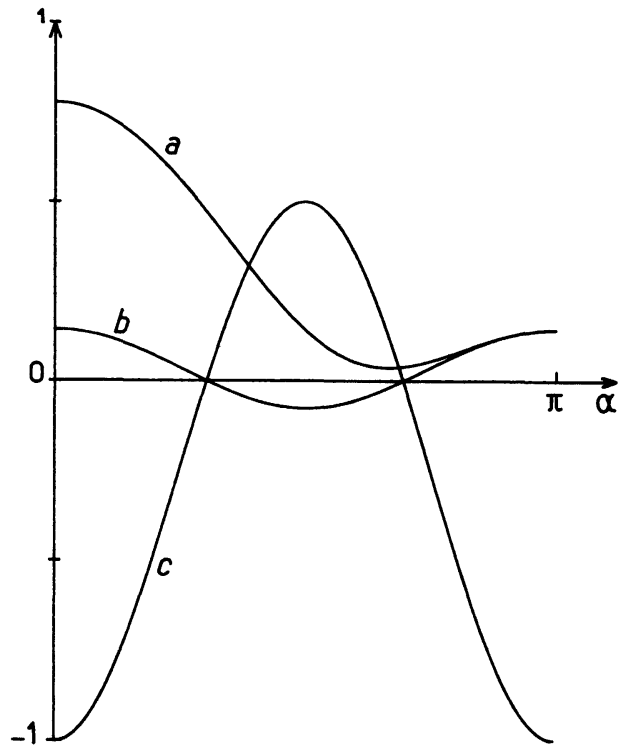

Fig. 1. - Variation of the polarization rate $P$ with the rotation angle $\alpha$ between $0-\pi$. Curve a corresponds to the calculation with interference effect (Eq. 46), while curve b corresponds to the calculation without interference effect (Eq. 47). Curve c repressents the anisotropy parameter $\beta$ (Eq. 48).

We may now calculate the radiated intensity for any polarization following the same lines as in reference 10 . We must rotate the molecular frame by an angle $\alpha$ around $\mathbf{J}$, taking $\mathbf{J}$ perpendicular to the internuclear axis. This involves an error of the order of $1 / J$, negligible when $J \gg 1$. We define $\mathbf{D}_{\mathrm{i}}$ and $\mathbf{D}_{\mathrm{f}}$, being the initial $\langle\mathrm{i}|\mathbf{D}| \mathrm{d}\rangle$ and final $\langle\mathrm{d}|\mathbf{D}| \mathrm{f}\rangle$ transition dipole moments, respectively. The calculation appears therefore to be purely geometrical. The intensity emitted with polarization $\mathbf{e}_{\mathrm{f}}$ appears to be proportional to $\left(D_{\mathrm{f}} \cdot \mathbf{e}_{\mathrm{f}}\right)^{2}$ (see Fig. 2). We must average over the angles $\theta, \varphi$ the excitation probability, which is given by

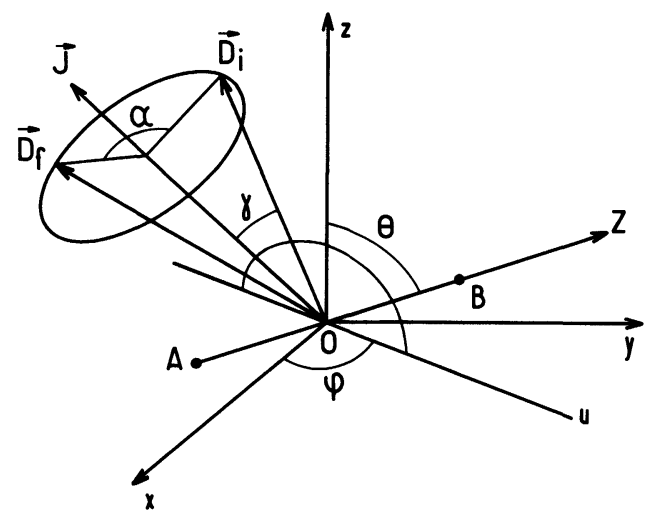

Fig. 2. - In the fixed laboratory frame Oxyz, the molecular axis $\mathrm{AB}=\mathrm{OZ}$ points initially in the direction $\theta, \phi$. The initial dipole moment $\mathrm{D}_{\mathrm{i}}$ is in the plane $\mathrm{O} z, \mathrm{OZ}$ perpendicular to $\mathrm{OZ}$. The total angular momentum $\mathbf{J}$ is taken perpendicular to $\mathrm{OZ}$, the angle between $\mathbf{D}_{i}$ and $\mathbf{J}$ is $\gamma$. After rotating of an angle $\alpha$ around $\mathbf{J}$, the dipole moment becomes $\mathbf{D}_{\mathrm{f}}$. 
$\sin ^{2} \theta \mathrm{d} \Omega$, and on the angle $\gamma\left(\right.$ angle between $\mathbf{J}$ and $\mathbf{D}_{\mathbf{i}}$ ) which is equiprobable between 0 and $2 \pi$. For excita- tion polarization along $\mathrm{OZ}$, the intensities emitted are :

$$
\begin{aligned}
& I_{\|}=I_{z}=\left[\sin \theta\left(\cos ^{2} \gamma+\sin ^{2} \gamma \cos \alpha\right)+\cos \theta \cos \gamma \sin \alpha\right]^{2} \\
& I_{\perp}=I_{x}=\left[\cos \varphi\left(\sin \gamma \sin \alpha \sin \theta-\cos \theta\left(\cos ^{2} \gamma+\sin ^{2} \gamma \cos \alpha\right)\right)+\sin \varphi \cos \gamma \sin \gamma(1-\cos \alpha)\right]^{2}
\end{aligned}
$$

which after averaging become :

$$
\begin{aligned}
& I_{\|}=(4 \pi / 15)\left(4+2 \cos \alpha+2 \cos ^{2} \alpha\right) \\
& I_{\perp}=(4 \pi / 15)\left(3-\cos \alpha-\cos ^{2} \alpha\right)
\end{aligned}
$$

leading to a polarization rate in accordance with equation 46. A simple, examination of the figure 2 proves that a rotation of $\pi$ decreases $I_{\|}$, this explains why $P$ involves directly. $\cos \alpha$. It is obvious on the other hand that a rotation of $\pi$ does not change the fragment distribution and thus the $\beta$ parameter depends on $\cos ^{2} \alpha$.

\section{Discussion of the $\mathrm{Ca}_{2}$ photodissociation experiment.}

Before discussing the main results of the experiment [10], let us present the electronic states involved in the process :

5.1 $\mathrm{Ca}_{2}$ MOleCular States. - Theoretical data on the electronic states of $\mathrm{Ca}_{2}$ is scarce. Only a few states have been calculated by Jones [20]. Detailed results exist for $\mathrm{Mg}_{2}$ [21] and $\mathrm{Zn}_{2}$ [22] which belong to the same family. In table $\mathrm{V}$, we present the asymptotic singlet states of $\mathrm{Ca}_{2}$, which are quite numerous. Among these states, detailed spectroscopic studies

Table V. - Asymptotic states of $\mathrm{Ca}_{2}$. For each disso-

\begin{tabular}{|c|c|c|c|}
\hline A & B & $\begin{array}{l}\text { Energy } \\
\left(\mathrm{cm}^{-1}\right)\end{array}$ & Molecular states \\
\hline- & - & - & - \\
\hline${ }^{1} \mathbf{S}$ & ${ }^{1} \mathrm{~S}$ & 0 & ${ }^{1} \Sigma_{\mathrm{g}}^{+}$ \\
\hline${ }^{1} S$ & ${ }^{3} \mathrm{P}$ & 15300 & 4 Triplet states \\
\hline${ }^{1} S$ & ${ }^{3} \mathrm{D}$ & 20350 & 6 Triplet states \\
\hline${ }^{1} \mathrm{~S}$ & ${ }^{1} \mathrm{D}$ & 21850 & $\left\{\begin{array}{l}{ }^{1} \Sigma_{\mathrm{q}}^{+} \cdot{ }^{1} \Sigma_{\mathrm{u}}^{+},{ }^{1} \Pi_{\mathrm{g}},{ }^{1} \Pi_{\mathrm{u}}, \\
{ }^{1} \Delta_{\mathrm{g}}, \Delta_{\mathrm{u}}\end{array}\right.$ \\
\hline${ }^{1} S$ & ${ }^{1} \mathbf{P}$ & 23652 & ${ }^{1} \Sigma_{\mathrm{g}}^{+},{ }^{1} \Sigma_{\mathrm{u}}^{+},{ }^{1} \Pi_{\mathrm{g}}{ }^{1} \Pi_{\mathrm{u}}$ \\
\hline${ }^{3} \mathbf{P}$ & ${ }^{3} \mathbf{P}$ & 30600 & $\left\{\begin{array}{l}{ }^{1} \Sigma_{\mathrm{g}}^{+}(2),{ }^{1} \Sigma_{\mathrm{u}}^{-},{ }^{1} \Pi_{\mathrm{g}}{ }^{1} \Pi_{\mathrm{u}} \\
{ }^{1} \Delta_{\mathrm{g}} 6 \text { Triplet and } \\
6 \text { Quintet states }\end{array}\right.$ \\
\hline $\mathrm{Ca}^{+2} \mathrm{~S}$ & $\mathrm{Ca}^{-2} \mathrm{P}\left({ }^{*}\right)$ & & $\left\{\begin{array}{l}{ }^{1} \Sigma_{\mathrm{g}}^{+},{ }^{1} \Sigma_{\mathrm{u}}^{+},{ }^{1} \Pi_{\mathrm{g}}{ }^{1} \Pi_{\mathrm{u}} \\
4 \text { Triplet states }\end{array}\right.$ \\
\hline
\end{tabular}
ciation limit, this table gives only the singlet states. Energy scale zero correspond to two ground states atoms (and not to the bottom of $X^{1} \Sigma_{\mathbf{g}}^{+}$well).

(*) Although $\mathrm{Ca}^{-}$may not be stable, this ionic configuration may contribute at short internuclear distances as it is the case for $\mathrm{Mg}_{2}$ [21]. exist only for the ground state $\mathrm{X}^{1} \Sigma_{\mathrm{g}}^{+}$and the $\mathrm{A}^{1} \Sigma_{\mathrm{u}}^{+}$, dissociating into ${ }^{1} \mathrm{~S}+{ }^{1} \mathrm{D}[23,24]$ (although some disagreement about what is the ground state exists [25]). Some work has also been done on $\mathrm{Ca}_{2}$ isolated in rare gas matrices at low temperature [26, 27]. In one of these [27] the $\mathrm{X}^{1} \Sigma_{\mathrm{g}}^{+} \rightarrow{ }^{1} \Pi_{\mathrm{u}}$ (dissociating into ${ }^{1} \mathrm{~S}+{ }^{1} \mathrm{P}$ ) transition has been observed.

Fortunately, in our problem most of these states are irrelevant. The dissociative excited state in the experiment gives one atom in the ${ }^{1} \mathrm{P}$ state, since we observe the fluorescence. Moreover, the initial state of the system is the ground state of the molecule $\mathrm{X}^{1} \Sigma_{\mathrm{g}}^{+}$, since there is no possible excitation mechanism in the oven or in the molecular beam. Krypton laser photons were used $(\lambda=406$ or $413 \mathrm{~nm}$, corresponding to 24579 or $\left.24178 \mathrm{~cm}^{-1}\right)$. This is very close to the excitation energy of the ${ }^{1} \mathrm{P}$ state of $\mathrm{Ca}\left(E_{1 \mathrm{p}}-E_{1 \mathrm{~s}}=\right.$ $23652 \mathrm{~cm}^{-1}$ [28]). Therefore the other $\mathrm{Ca}$ atom produced by photodissociation is necessarily in its ground state ${ }^{1} \mathrm{~S}_{0}$. As a consequence, we need only to consider the states dissociating into ${ }^{1} \mathrm{~S}+{ }^{1} \mathrm{P}$ and in addition, due to the $\mathrm{u} \leftarrow \mathrm{g}$ selection rule for electric dipole transitions, only the ${ }^{1} \Sigma_{\mathrm{u}}^{+}$and the ${ }^{1} \Pi_{\mathrm{u}}$ states can be reached by optical excitation.

As evidenced in the case of $\mathrm{Mg}_{2}$, the ${ }^{1} \Pi_{\mathrm{u}}$ potential curve interacts at short internuclear distances with an ionic curve of the same symmetry. More generally, in $\mathrm{Ca}_{2}$, the states dissociating into ${ }^{1} \mathrm{~S}+{ }^{1} \mathbf{P}$ may interact also with states arising from ${ }^{1} S+{ }^{1} D$ or ${ }^{3} \mathrm{P}+{ }^{3} \mathrm{P}$.

In particular, this may be the case for the $\mathrm{A}^{1} \Sigma_{\mathrm{u}}^{+}$ state of $\mathrm{Ca}_{2}$, which dissociates adiabatically into ${ }^{1} S+{ }^{1} D$ [24] but which could correlate diabatically to ${ }^{1} \mathrm{~S}+{ }^{1} \mathrm{P}$ as suggested by the figure 3 .

For large internuclear distances $R \gtrsim 5 \AA$ the potential curves of the states dissociating into ${ }^{1} \mathrm{~S}+{ }^{1} \mathrm{P}$ are dominated by the long-range dipole resonance interaction [29]. The resulting potential curves for the ${ }^{1} \Pi_{\mathrm{u}}$ and ${ }^{1} \Sigma_{\mathrm{u}}^{+}$states are given by :

$$
\begin{aligned}
V_{1_{\Pi_{\mathrm{u}}}}(R) & =E_{1 \mathrm{~S}}+E_{1_{\mathrm{p}}}+\frac{\mu_{\mathrm{m}}^{2}}{R^{3}}= \\
& =23652+\frac{2.64 \times 10^{5}}{R^{3}}\left(\mathrm{~cm}^{-1}\right) \\
V_{1_{\Sigma^{+}}}(R) & =E_{1 \mathrm{~S}}+E_{1 \mathrm{P}}-\frac{2 \mu_{\mathrm{m}}^{2}}{R^{3}}= \\
& =23652-\frac{5.28 \times 10^{5}}{R^{3}}\left(\mathrm{~cm}^{-1}\right)(R \text { in } \AA) .
\end{aligned}
$$




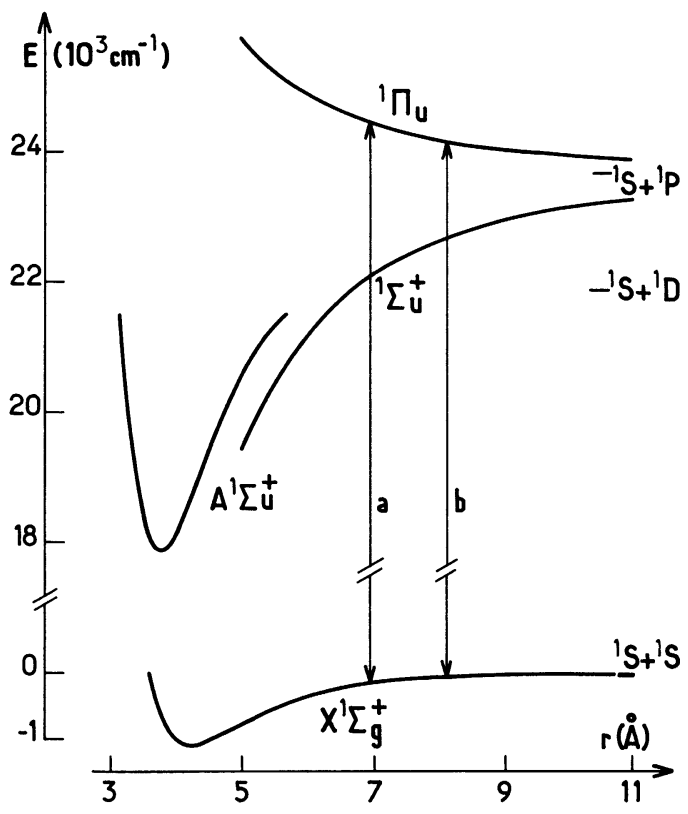

Fig. 3. - Potential curves for the $\mathrm{X}^{1} \Sigma_{\mathrm{g}}^{+}, \mathrm{A}^{1} \Sigma_{\mathrm{u}}^{+},{ }^{1} \Sigma_{\mathrm{u}}^{+}$ and ${ }^{1} \Pi_{\mathrm{u}}$ states discussed in the text. From the ${ }^{1} S+{ }^{1} \mathrm{D}$ limit, ${ }^{1} \Sigma_{\mathrm{u}, \mathrm{g}}^{+}{ }^{1} \Pi_{\mathrm{u}, \mathrm{g}},{ }^{1} \Delta_{\mathrm{u}, \mathrm{g}}$ states arise. The energy scale is in unit of $10^{3} \mathrm{~cm}^{-1}$. The a (b) arrow corresponds to the vertical transition with an energy equal to the energy of the laser photons $406(413) \mathrm{nm}$. This graphical representation clearly exhibits the fact that the present dissociation experiment tests only the relatively long range part of the $\mathrm{X}$ and ${ }^{1} \Pi_{\mathrm{u}}$ potential curves.

The value for $\mu_{\mathrm{m}}^{2}$ is deduced from the ${ }^{1} \mathrm{P}-{ }^{1} \mathrm{~S}$ transition coefficient [30]. We have neglected higher order terms in the $C_{n} R^{-n}$ expansion. This means that the typical error is comparable to the $C_{6} R^{-6}$ term in the ground state, which is close to $250 \mathrm{~cm}^{-1}$ for $R=6 \AA$, while the $\mu_{\mathrm{m}}^{2} / R^{3}$ term is close to $1200 \mathrm{~cm}^{-1}$. This provides an idea of the limited accuracy of this evaluation. We have plotted in figure 3 the resulting ${ }^{1} \Pi_{\mathrm{u}}$ and ${ }^{1} \Sigma_{\mathrm{u}}^{+}$ potential curves together with the RKR curves of the $\mathrm{A}^{1} \Sigma_{\mathrm{u}}^{+}$and $\mathrm{X}^{1} \Sigma_{\mathrm{g}}^{+}$states [24]. The fact that the $\mathrm{A}^{1} \Sigma_{\mathrm{u}}^{+}$ and ${ }^{1} \Sigma_{u}^{+}\left({ }^{1} S+{ }^{1} \mathrm{P}\right)$ are close together in the region $R=5 \AA$, supports the idea of adiabatic correlation of the A state into ${ }^{1} \mathrm{~S}+{ }^{1} \mathrm{P}$ fragments.

In our case, we are not directly concerned by the ${ }^{1} \Sigma_{u}^{+}$ state. If the dissociative state reached by the optical excitation was of that symmetry, there would be no way of reconciling the experimental and theoretical polarization ratios [10].

The strongly repulsive character of the ${ }^{1} \Pi_{u}$ state is a favourable circumstance : as the laser photons are close to the energy of the ${ }^{1} \mathrm{P}-{ }^{1} \mathrm{~S}$ transition, the experiment samples only a small range of the ${ }^{1} \Pi_{\mathrm{u}}$ curve :

$$
V_{1_{\Pi_{\mathrm{u}}}}(R)<R_{\mathbf{1}_{\mathrm{S}}}+E_{1_{\mathrm{P}}}+h v
$$

which gives $R \geqslant 6.6 \AA$ for the $406 \mathrm{~nm}$ photon. We hope that in this range the potential curve given by equation 52 is reasonably good.

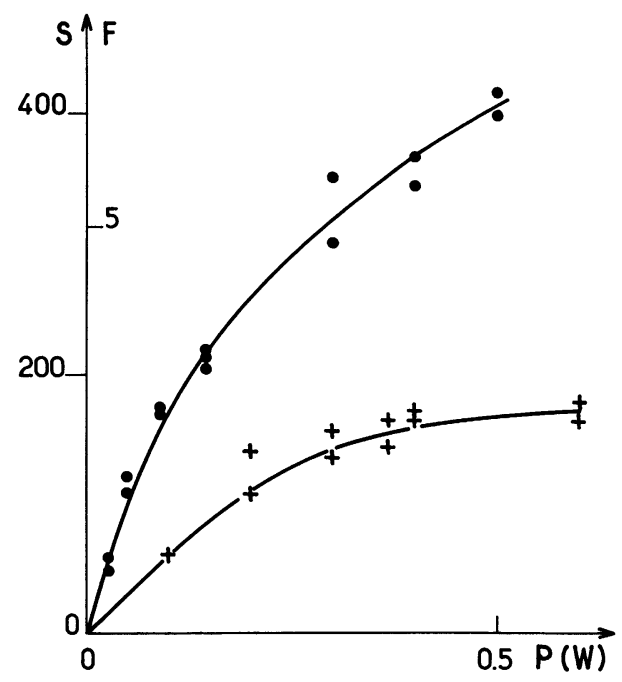

Fig. 4. - The atomic fluorescence signal at $422.7 \mathrm{~nm}$ expressed by the number $S$ of photons detected per second is plotted as a function of the laser power $P$ (in watt), for the two excitation wavelengths : $406 \mathrm{~nm}$ (dots) and $413 \mathrm{~nm}$ (crosses). The corresponding dissociation fraction of ground state molecules $F$ is also given (expressed in \%). The curves are just intended for visual aid.

5.2 BRIEF RECALL OF THE EXPERIMENTAL CONDITIONS AND RESULTS. - The experiment has already been described elsewhere [10]. In brief, $\mathrm{Ca}_{2}$ molecules were produced in an oven (temperature close to $1000 \mathrm{~K}$ ) and formed an effusive molecular beam (containing mainly $\mathrm{Ca}$ atoms). The signal was clearly due to $\mathrm{Ca}_{2}$ as it was linearly dependent on the calculated $\mathrm{Ca}_{2}$ density. The molecules interacted with the laser beam about $10^{-4} \mathrm{~s}$ after leaving the oven, which insures that only stable ground state molecules were excited. The fraction of dissociated molecules was measured through an absolute calibration of the detection apparatus $[10,31]$. The fraction $F$ was

$$
\begin{aligned}
& F \cong 0.04 \text { for } \lambda=406 \mathrm{~nm} \\
& F \simeq 0.02 \text { for } \lambda=413 \mathrm{~nm}
\end{aligned}
$$

using in both cases a beam with a $0.2 \mathrm{~W}$ power focused on a beam waist of radius $\omega_{0} \simeq 28 \mu$. The atomic fluorescence intensity saturates when the laser power increases as shown in figure 4. The polarization $P$ of the ${ }^{1} \mathrm{P}_{-}{ }^{1} \mathrm{~S} \mathrm{Ca}$ fluorescence light was measured :

$$
\begin{array}{ll}
P=0.64 \pm 0.01 & \text { for } \lambda=406 \mathrm{~nm} \\
P=0.68 & \text { for } \lambda=413 \mathrm{~nm} .
\end{array}
$$

The presence of some stray light in the second case made this measurement more uncertain. As the detection apparatus is using a wide aperture lens $(f / 0.8)$, the polarization ratio $P$ is reduced. This reduction appears to be $0.02-0.03$ according to the formulae of Zinsli [32]. 
5.3 ANALYSIS OF THE $\mathrm{Ca}_{2} \mathrm{X}{ }^{1} \Sigma_{\mathrm{g}}^{+}-{ }^{1} \Pi_{\mathrm{u}}$ PHOTODISSOCIATION PROCESS. - The first point to be made is that due to the coherence effect between $\Lambda=1$ and $\boldsymbol{\Lambda}=-1$ in the ${ }^{1} \Pi_{\mathrm{u}}$ excited state, we expect a maximum polarization rate $P=0.78$, which is larger than the observed values. On the other hand, if the coherence effects are not taken into account the polarization would be $P=0.14$ which is clearly in contradiction with the experimental findings. The explanation for the difference between our maximum predicted value of 0.78 and the experimental results is to be searched in various effects, the first of them being molecular rotation. We must therefore determine the value of the rotational quantum number of the states which are photodissociated.

The oven temperature is close to $1000 \mathrm{~K} ; \mathrm{Ca}_{2}$ thermal population is spread ovel all the rovibrational levels of the ground state, as the well depth of this state is only $1095 \mathrm{~cm}^{-1}$ (comparable to $k T \sim 700 \mathrm{~cm}^{-1}$ ). The photodissociation signal is therefore the sum of the signals arising from each $v_{\mathrm{i}} J_{\mathrm{i}}$ level of the ground state satisfying :

$$
E_{v_{\mathrm{i}} \mathrm{J}_{\mathrm{i}}}+h v_{\mathrm{i}} \geqslant E_{1 \mathrm{~S}}+E_{1 \mathrm{p}} .
$$

This equation gives the thermodynamic threshold for dissociation which is given in table VI. The threshold is rather low, and on this basis we can assert that a very large fraction of the ground state population can be dissociated. However, the rate of transition from the ground state level $\mathrm{X}_{v_{\mathrm{i}} J_{\mathrm{i}}}$ to the ${ }^{1} \Pi_{\mathrm{u}} \varepsilon_{\mathrm{d}} J_{\mathrm{d}}$

Table VI. - For the two laser wavelengths used in the $\mathrm{Ca}_{2}$ experiment, we give the wavenumber $v$, the thermodynamic threshold and the corresponding dissociation fraction $F^{\text {th }}$. The pseudo crossing point $r^{*}$, the potential energy in the $X$ state for this $r$ value are given. The dissociation fraction $F^{\mathrm{FC}}$ is deduced from the Franck-Condon condition (Eq. 59). The maximum $J$ value, $J_{\mathrm{m}}$, and root mean square value, $J^{\mathrm{RMS}}$ are calculated, as well as the corresponding rotation angle $\alpha$ and the polarization rate $P$.
$\lambda(\AA)$
4131.33
$v\left(\mathrm{~cm}^{-1}\right)$
24199
4067.37
24579

Thermodynamic

threshold $\langle E(v, J)\rangle$

549

$$
\begin{gathered}
\left(\mathrm{cm}^{-1}\right) \\
F^{\text {th }} \\
r^{*}(\AA) \\
V_{\mathrm{X}}\left(r^{*}\right)\left(\mathrm{cm}^{-1}\right) \\
F^{\mathrm{FC}} \\
\left\{\begin{array}{l}
J_{\mathrm{m}} \\
\alpha_{\mathrm{m}}(\mathrm{rad}) \\
P
\end{array}\right. \\
\left\{\begin{array}{l}
J^{\mathrm{RMS}} \\
\alpha^{\mathrm{RMS}} \\
P
\end{array}\right.
\end{gathered}
$$

1044 level is dominated by the Franck-Condon density (neglecting the $R$ variation of the transition moment)

$$
\left|\left\langle\chi_{v_{1} J_{i}} \mid \chi_{\varepsilon_{\mathrm{d}} J_{\mathrm{d}}}\right\rangle\right|^{2} \text { with } \varepsilon_{\mathrm{d}}=E_{v_{\mathrm{i}} J_{\mathrm{i}}}+h v_{\mathrm{i}} .
$$

Such a Franck-Condon density can be investigated approximatively using semiclassical arguments [33]. The Franck-Condon density is large only around the pseudo-crossing $R^{*}$ defined by

$$
V_{\mathbf{X}^{1} \Sigma^{+}}\left(R^{*}\right)+h v_{\mathbf{i}}=V_{{ }^{1} \Pi_{\mathbf{u}}}\left(R^{*}\right)
$$

with the condition that this point is in the allowed classical region for vibrational motion of the level $v_{\mathrm{i}} J_{\mathrm{i}}$ (and also for the level $E J_{\mathrm{d}}$ ). This additional condition means that only those levels $v_{\mathrm{i}} J_{\mathrm{i}}$ for which

$$
E_{v_{\mathbf{i}} J_{\mathbf{i}}} \geqslant V_{\mathbf{X}^{1} \Sigma_{\mathbf{g}}}\left(R^{*}\right)+\frac{\hbar^{2}}{2 \mu R^{* 2}} J_{\mathrm{i}}\left(J_{\mathrm{i}}+1\right)
$$

can be easily dissociated. For levels with an energy slightly lower, the Franck-Condon density is not zero but decreases exponentially. Values of $R^{*}$ and $V_{\mathbf{X}^{1} \Sigma_{\mathbf{g}}^{+}}\left(R^{*}\right)$ appear in table VI. This new condition is much stronger than the thermodynamic one. The corresponding fractions of dissociation are also given in table VI and they are clearly in agreement with the experimental values at saturation as they can be deduced from figure 4.

To calculate the polarization ratio of the fluorescence, we need the value of the rotation angle $\alpha$ for each level $v_{\mathrm{i}} J_{\mathrm{i}}$ which contributes to the photodissociation signal (i.e. which fulfills condition (59)). Limiting the calculation to the term linear in $J_{i}$, we get by a classical calculation [34]

$$
\alpha=\beta\left[\frac{\hbar^{2} J_{\mathrm{i}}\left(J_{\mathrm{i}}+1\right)}{2 \mu R_{0}^{2}\left[V_{{ } \Pi_{\mathrm{u}}}\left(R_{0}\right)-V_{{ } \Pi_{\mathrm{u}}}(\infty)\right]}\right]^{1 / 2}
$$

where $R_{0}$ is the inner turning point of the classical motion for the level ${ }^{1} \Pi_{\mathrm{u}} E J_{\mathrm{d}}$

$$
\left(V_{{ }^{1} \Pi_{\mathrm{u}}}\left(R_{0}\right)+\frac{\hbar^{2}}{2 \mu R_{0}^{2}} J_{\mathrm{d}}\left(J_{\mathrm{d}}+1\right)=E\right),
$$

$R_{0}$ is close to $R^{*}$. The constant $\beta$ is given by $\int_{0}^{1}\left(1-u^{3}\right)^{-1 / 2} \mathrm{~d} u \cong 1.4$. Using this formula, we can calculate for each level $\alpha$ and the value of $P$. We give as an example the maximum value of $J$, denoted $J_{\mathrm{m}}$, and the corresponding value of $\alpha$ and $P$. We need to calculate an average value of $P$. As $\alpha$ remains small and in that case $P \sim 7 / 9-10 \alpha^{2} / 27$, we have calculated the root mean square value of $\alpha$, $\alpha_{\mathrm{rms}}$, the weight of the level $v_{\mathrm{i}} J_{\mathrm{i}}$ being

$$
\left(2 J_{i}+1\right) \exp \left[-E_{v_{1} J_{i}} / k T\right],
$$

and the sum involves levels which satisfy equation 59 only. The polarization ratio corresponding to $\alpha_{\mathrm{rms}}$ 
is also given in table VI. We conclude from these values that the rotation is small and does not change significantly the polarization of the fluorescence.

The second possible reason for the smaller polarization ratio observed as compared with the maximum theoretical value, is the saturation effect. On figure 4 we show the saturation of the atomic fluorescence signal as a function of the laser power. This saturation corresponds to a depletion of the ground state levels (at least for the ones connected to the ${ }^{1} \Pi_{\mathrm{u}}$ state by large Franck-Condon factors). It is simple to study this saturation effect. The rate of depletion $\gamma\left(v_{\mathrm{i}} J_{\mathrm{i}} M_{\mathrm{i}}\right)$ of the level $\mathrm{X}_{v_{\mathrm{i}} \mathrm{J}_{\mathrm{i}} M_{\mathrm{i}}}$ can be deduced from the Fermi Golden Rule [35]

$$
\begin{aligned}
& \gamma\left(v_{\mathrm{i}} J_{\mathrm{i}} M_{\mathrm{i}}\right)= \\
& =\frac{2 \pi}{\hbar} \sum_{J_{\mathrm{d}} M_{\mathrm{d}}}\left|\left\langle\mathrm{X}_{v_{\mathrm{j}} J_{\mathrm{i}} M_{\mathrm{i}}}\left|D_{\mathrm{z}} E_{\mathrm{z}}\right|^{1} \Pi_{\mathrm{u}} E_{\mathrm{d}} J_{\mathrm{d}} M_{\mathrm{d}}\right\rangle\right|^{2}
\end{aligned}
$$

$E_{\mathrm{z}}$ is the laser electric field, assumed to be linearly polarized. The validity of this formula has been studied in detail [36]; it depends on the width $\Delta \varepsilon$ of the Franck-Condon density $\left|\left\langle\chi_{v_{1} J_{\mathrm{i}}} \mid \chi_{\varepsilon J \mathrm{~d}}\right\rangle\right|^{2}$ considered as a function of $\varepsilon$. Using semiclassical arguments [33], we can estimate this width to be $\Delta \varepsilon \sim 10^{2} \mathrm{~cm}^{-1}$ and the maximum value of this Franck-Condon density to be $\sim 10^{-2} \mathrm{~cm}$. The validity condition is that this width should be large compared to the Rabi frequency $\hbar \Omega_{\mathrm{R}}=D_{\mathrm{z}} E_{\mathrm{z}}$.
The electric dipole moment is easy to evaluate, using molecular wavefunctions obtained by symmetrization of atomic wavefunctions,

$$
\begin{aligned}
& \left|\left\langle\mathrm{X}^{1} \Sigma_{\mathrm{g}}^{+}\left|D_{-1}^{1}\right|^{1} \Pi_{\mathrm{u}}, \Lambda=1\right\rangle\right|^{2}= \\
& \quad=\frac{2}{3}\left|\left\langle{ }^{1} \mathrm{~S}_{0}\left\|D^{1}\right\|{ }^{1} \mathrm{P}\right\rangle\right|^{2} \simeq 16.2 \mathrm{a} \text {.u. }
\end{aligned}
$$

For a laser power $P=0.2 \mathrm{~W}$ and a beam waist radius $\omega_{0}=28 \mu$ the rms laser electric field is

$$
E=\left[P /\left(\pi \omega_{0}^{2} \varepsilon_{0} c\right)\right]^{1 / 2}=1.7 \times 10^{5} \mathrm{~V} / \mathrm{m}
$$

and the Rabi frequency is close to $0.3 \mathrm{~cm}^{-1}$, clearly negligible when compared to $\Delta \varepsilon \simeq 10^{2} \mathrm{~cm}^{-1}$. The resulting excitation rate for a $\mathrm{Ca}_{2}$ molecule is, for the maximum value of the Franck-Condon density, $\gamma \sim 10^{9} \mathrm{~s}^{-1}$. As the time $\tau$ necessary to cross the beam at the average speed $v$ (for a molecular beam at $1000 \mathrm{~K}, v \sim 600 \mathrm{~m} / \mathrm{s}), \tau=10^{-7} \mathrm{~s}$, the excitation is clearly saturated for the levels connected by large Franck-Condon densities. In the present case the small value of the Rabi frequency insures that the molecules can be excited but cannot radiate back to the ground state by stimulated emission.

Therefore using rate equations it is possible to calculate, in the presence of saturation the atomic light intensity as a function of the polarization $\mathbf{e}_{\mathrm{f}}$

$$
\begin{aligned}
I\left(\mathbf{e}_{\mathrm{f}}\right)=\sum_{v_{\mathrm{f}} J_{\mathrm{i}} M_{\mathrm{i}}} \eta_{0}\left(v_{\mathrm{i}} J_{\mathrm{i}} M_{\mathrm{i}}\right)\left(1-\exp \left[-\gamma\left(v_{\mathrm{i}} J_{\mathrm{i}} M_{\mathrm{i}}\right) \tau\right]\right) / \gamma\left(v_{\mathrm{i}} J_{\mathrm{i}} M_{\mathrm{i}}\right) \times \\
\quad \times \sum_{\varepsilon_{\mathrm{f}} J_{\mathrm{f}} M_{\mathrm{f}}}\left|\sum_{\varepsilon_{\mathrm{d}} J_{\mathrm{d}} M_{\mathrm{d}}} \frac{\left\langle v_{\mathrm{i}} J_{\mathrm{i}} M_{\mathrm{i}}\left|\mathbf{D}_{\mathrm{i}} \cdot \mathbf{e}_{\mathrm{i}}\right| \varepsilon_{\mathrm{d}} J_{\mathrm{d}} M_{\mathrm{d}}\right\rangle\left\langle\varepsilon_{\mathrm{d}} J_{\mathrm{d}} M_{\mathrm{d}}\left|\mathbf{D}_{\mathrm{f}} \cdot \mathbf{e}_{\mathrm{f}}\right| \varepsilon_{\mathrm{f}} J_{\mathrm{f}} M_{\mathrm{f}}\right\rangle}{E-\varepsilon_{\mathrm{d}}+i \Gamma_{\mathrm{d}}}\right|^{2} .
\end{aligned}
$$

It is clear that this equation behaves correctly for the non-saturated limit. In the presence of saturation, it is no longer possible to sum over the magnetic quantum numbers $M$ as we did in section 2 . Rather than evaluating numerically this equation, we may try to understand its consequence in a particular case. The excitation rate $\gamma_{v_{i} J_{i} M_{i}}$ depends on $M_{i}$, it is the quantum analog of the classical excitation probability as a function of the orientation of the internuclear axis [10]. In the presence of saturation all the molecules from the ground state level $v_{\mathrm{i}} J_{\mathrm{i}}$ are transferred, whatever their $\boldsymbol{M}_{\mathrm{i}}$ values are into the excited state. This means classically that the excited molecular axis becomes isotropically distributed. In the absence of molecular rotation during the dissociation, this leads to a polarization ratio

$$
P=0.6
$$

in the case of the first geometry considered in table II which corresponds to that of the experiment on $\mathrm{Ca}_{2}$. We therefore believe that saturation has an important rôle in the decrease of the polarization ratio from the maximum theoretical value of 0.78 to the observed 0.64 . A detailed calculation of this effect should consider the fact that as the Franck-Condon densities vary greatly, the signal is the sum of signals corresponding to different states of saturation.

Another possible source of depolarization could be radiation trapping, i.e., the re-absorption and reemission of the $\mathrm{Ca}\left({ }^{1} \mathrm{P}\right)$ fluorescence in the beam. Fluorescence trapping is well known to reduce the polarization rate on resonance lines. However, in the condition of the experiment we believe that this effect should be minor, although we cannot dismiss it altogether.

From the discussion above, we conclude that the polarization ratio observed experimentally in $\mathrm{Ca}_{2}$ can be understood if :

i) The coherence effects due to interference between different $\Lambda= \pm 1$ states in the intermediate manifold are taken into account. 
ii) The depolarization effects (instrumental, rotation, saturation and possibly radiation trapping) are considered. We believe that the most important source of depolarization comes from the saturation effect.

5.4 SOME ADDITIONAL EFFECTS. - We would like to discuss here some other effects that could play a rôle in other cases but which seem negligible here :

i) Alignment of molecules in the beam. Supersonic beams have been shown to produce aligned molecules [37]. The beam used in the $\mathrm{Ca}_{2}$ experiment was of the effusive type. This was verified in detail [31].

ii) Non adiabatic effects in the photodissociation. As suggested in reference 38 when the ${ }^{1} \Pi_{\mathrm{u}}-{ }^{1} \Sigma_{\mathrm{u}}^{+}$ separation becomes comparable to the non-adiabatic Coriolis coupling $-\frac{\hbar^{2}}{2 \mu R^{2}}\left(J_{+} L_{-}+J_{-} L_{+}\right)$between these states, the rotation of the molecular axis is no longer followed by a rotation of the electronic cloud. This effect must reduce the importance of the depolarization due to rotation. A detailed analysis of this effect has been performed by Grosser [39]. The distance at which this decoupling occurs is given by (applying Eq. 12 of Ref. 39)

$$
V_{1_{\Pi}}(R)-V_{1_{\Sigma}}(R) \sim \frac{\hbar v}{R}
$$

where $v$ is the relative speed of the Ca atoms. Applied to our case we get for the decoupling distance :

$$
R \sim 200 \AA \text { for } v \sim 500 \mathrm{~m} / \mathrm{s} .
$$

This value is sufficiently large so that the molecular rotation is almost completed; the molecular axis has made its rotation through the angle $\alpha$ within a few percent. We then conclude that in the present case the effect of this coupling is negligible.

iii) Interference between dissociation paths going through electronic states of different symmetry [40]. In our case we should have

$$
\mathrm{X}^{1} \Sigma_{\mathrm{g}}^{+} \longrightarrow{ }^{1} \Pi_{\mathrm{u}} \longrightarrow \mathrm{X}^{1} \Sigma_{\mathrm{u}}^{+} \Sigma_{\mathrm{g}}^{+}
$$

This interference is important if the Franck-Condon densities for these two processes are of comparable magnitude for the same initial levels and the same photon energy. This is not the case for $\mathrm{Ca}_{2}$ since the ${ }^{1} \Pi_{\mathrm{u}}$ and the ${ }^{1} \Sigma_{\mathrm{u}}^{+}$potential curves are very different.

iv) Electric dipole approximation. This approximation was used through all this work, without any questioning of its validity even if the molecule is as large as $10^{4}-10^{5} \AA$, i.e. larger than the wavelength $\lambda$ emitted. This is surely correct for heteronuclear molecules which can be described as one atom excited and one in the ground state, the last being only a spectator in the radiation process. For an homonuclear molecule, as soon as the electronic overlap becomes negligible, one can assume a dipole on each atom, both emitting coherently as long as $R \ll \lambda$. The emission rate is 2 times the atomic emission rate and it drops back to the atomic emission rate for large $R(R \gtrsim \lambda)$. This effect has fortunately no direct consequence on the polarization of the light emitted (it has an indirect effect through the fact that more light is emitted during the dissociation before rotation).

v) The theory presented here is for atomic fragments without nuclear spin. The presence of hyperfine structure can reduce the theoretical polarizations.

\section{Acknowledgments}

The authors would like to acknowledge A. Aspect, P. Grangier and G. Roger for their kindly communication of important experimental data and information. We would also like to thank J. Durup, C. Cohen-Tannoudji and J. Grosser for very helpful discussions, and the referee for his pertinent remarks.

\section{Appendix A.}

SUMMATION OVER MAGNETIC QUANTUM NUMBERS. - Using equations 7, 8 and 11, and the relationship [17] :

$$
\begin{aligned}
& \sum_{M_{\mathrm{d}}}(-1)^{J_{\mathrm{i}}-M_{\mathrm{i}}}\left(\begin{array}{ccc}
J_{\mathrm{i}} & 1 & J_{\mathrm{d}} \\
-M_{\mathrm{i}} & p & M_{\mathrm{d}}
\end{array}\right)(-1)^{J_{\mathrm{d}}-M_{\mathrm{d}}}\left(\begin{array}{ccc}
J_{\mathrm{d}} & 1 & J_{\mathrm{f}} \\
-M_{\mathrm{d}} & q & M_{\mathrm{f}}
\end{array}\right)= \\
& =\sum_{K Q}(2 K+1)(-1)^{J_{\mathrm{i}}-M_{\mathrm{i}}}\left(\begin{array}{ccc}
J_{\mathrm{i}} & K & J_{\mathrm{f}} \\
-M_{\mathrm{i}} & Q & M_{\mathrm{f}}
\end{array}\right)(-1)^{2 J_{\mathrm{d}}+Q}\left(\begin{array}{lll}
1 & 1 & K \\
p & q & Q
\end{array}\right) \times\left\{\begin{array}{lll}
1 & 1 & K \\
J_{\mathrm{f}} & J_{\mathrm{i}} & J_{\mathrm{d}}
\end{array}\right\}
\end{aligned}
$$

it is obtained :

$$
\frac{\mathrm{d} \sigma}{\mathrm{d} \Omega_{\mathrm{f}}}=2 \pi k_{\mathrm{i}} k_{\mathrm{f}}^{3} \sum_{M_{\mathrm{i}}} \sum_{\Omega_{\mathrm{f}} \varepsilon_{\mathrm{f}} J_{\mathrm{f}} M_{\mathrm{f}}}\left|\sum_{K Q} a_{K Q}(2 K+1)(-1)^{J_{\mathrm{i}}-M_{\mathrm{i}}}\left(\begin{array}{ccc}
J_{\mathrm{i}} & K & J_{\mathrm{f}} \\
-M_{\mathrm{i}} & Q & M_{\mathrm{f}}
\end{array}\right)\right|^{2}
$$


where :

$$
\begin{aligned}
& a_{K Q}=\sum_{\Omega_{\mathrm{d}} \varepsilon_{\mathrm{d}} \sigma_{\mathrm{d}}} \sum_{p, m, q, n}(-1)^{p+q+Q}\left(E-\varepsilon_{\mathrm{d}}+i \Gamma_{\mathrm{d}}\right)^{-1}\left(e_{\mathrm{i}}\right)_{p}^{1}\left(e_{\mathrm{f}}^{*}\right)_{q}^{1} \times \\
& \times\left(\begin{array}{lll}
1 & 1 & K \\
p & q & Q
\end{array}\right)\left\langle\Omega_{\mathrm{i}} v_{\mathrm{i}} J_{\mathrm{i}}\left|(D)_{m}^{1}\right| \Omega_{\mathrm{d}} \varepsilon_{\mathrm{d}} J_{\mathrm{d}}\right\rangle\left\langle\Omega_{\mathrm{d}} \varepsilon_{\mathrm{d}} J_{\mathrm{d}}\left|(D)_{n}^{1}\right| \Omega_{\mathrm{f}} \varepsilon_{\mathrm{f}} J_{\mathrm{f}}\right\rangle \\
& \times\left(2 J_{\mathrm{d}}+1\right)\left(2 J_{\mathrm{f}}+1\right)^{1 / 2}(-1)^{2 J_{\mathrm{d}}}\left\{\begin{array}{lll}
1 & 1 & K \\
J_{\mathrm{f}} & J_{\mathrm{i}} & J_{\mathrm{d}}
\end{array}\right\}(-1)^{J_{\mathrm{i}}-\Omega_{\mathrm{i}}}\left(\begin{array}{rll}
J_{\mathrm{i}} & 1 & J_{\mathrm{d}} \\
-\Omega_{\mathrm{i}} & m & \Omega_{\mathrm{d}}
\end{array}\right) \\
& \times(-1)^{J_{\mathrm{d}}-\Omega_{\mathrm{d}}}\left(\begin{array}{rrr}
J_{\mathrm{d}} & 1 & J_{\mathrm{f}} \\
-\Omega_{\mathrm{d}} & n & \Omega_{\mathrm{f}}
\end{array}\right) .
\end{aligned}
$$

Expanding the square in (A.2), and using the normalization of the $3-j$ coefficients, we get :

$$
\frac{\mathrm{d} \sigma}{\mathrm{d} \Omega_{\mathrm{f}}}=2 \pi k_{\mathrm{i}} k_{\mathrm{f}}^{3} \sum_{\Omega_{\mathrm{f}} \varepsilon_{\mathrm{f}} J_{\mathrm{f}}} \sum_{K Q}(2 K+1)\left|a_{K Q}\right|^{2}
$$

from which equation 13 can be easily written.

\section{Appendix B.}

AXIAL RECOIL APPROXIMATION. - In the axial recoil approximation, we can write from equation 13 :

$$
\begin{gathered}
\frac{\mathrm{d} \sigma}{\mathrm{d} \Omega_{\mathrm{f}}} \propto \sum_{J_{\mathrm{f}}}\left(2 J_{\mathrm{f}}+1\right)\left|\mathcal{F}_{K}\right|^{2} \mid \sum_{\Omega_{\mathrm{d}}} b_{\Omega_{\mathrm{d}}} \sum_{J_{\mathrm{d}}}(-1)^{2 J_{\mathrm{d}}}\left(2 J_{\mathrm{d}}+1\right) \times \\
\cdot \quad \times\left.\left\{\begin{array}{lll}
1 & 1 & K \\
J_{\mathrm{f}} & J_{\mathrm{i}} & J_{\mathrm{d}}
\end{array}\right\}\left(\begin{array}{ccc}
J_{\mathrm{i}} & 1 & J_{\mathrm{d}} \\
-\Omega_{\mathrm{i}} & m & \Omega_{\mathrm{d}}
\end{array}\right)(-1)^{J_{\mathrm{d}}-\Omega_{\mathrm{d}}}\left(\begin{array}{ccc}
J_{\mathrm{d}} & 1 & J_{\mathrm{f}} \\
-\Omega_{\mathrm{d}} & n & \Omega_{\mathrm{f}}
\end{array}\right)\right|^{2} \\
n=\Omega_{\mathrm{d}}-\Omega_{\mathrm{f}} \quad m=\Omega_{\mathrm{i}}-\Omega_{\mathrm{d}}
\end{gathered}
$$

where :

$$
b_{\Omega_{\mathrm{d}}}=\sum_{\varepsilon_{\mathrm{d}}}\left(E-\varepsilon_{\mathrm{d}}+i \Gamma_{\mathrm{d}}\right)^{-1}\left\langle\Omega_{\mathrm{i}} v_{\mathrm{i}} J_{\mathrm{i}}\left|(D)_{m}^{1}\right| \Omega_{\mathrm{d}} \varepsilon_{\mathrm{d}} J_{\mathrm{d}}\right\rangle\left\langle\Omega_{\mathrm{d}} \varepsilon_{\mathrm{d}} J_{\mathrm{d}}\left|(D)_{n}^{1}\right| \Omega_{\mathrm{f}} \varepsilon_{\mathrm{f}} J_{\mathrm{f}}\right\rangle .
$$

Using again the relation (A.1), written in the form :

$$
\begin{aligned}
& \sum_{J_{\mathrm{d}}}(-1)^{J_{\mathrm{d}}+K+\Omega_{\mathrm{d}}-\Omega_{\mathrm{f}}-\Omega_{\mathrm{i}}}\left(2 J_{\mathrm{d}}+1\right)\left\{\begin{array}{lll}
1 & 1 & K \\
J_{\mathrm{f}} & J_{\mathrm{i}} & J_{\mathrm{d}}
\end{array}\right\} \times \\
& \left(\begin{array}{rll}
J_{\mathrm{i}} & 1 & J_{\mathrm{d}} \\
-\Omega_{\mathrm{i}} & m & \Omega_{\mathrm{d}}
\end{array}\right)\left(\begin{array}{ccc}
J_{\mathrm{d}} & 1 & J_{\mathrm{f}} \\
-\Omega_{\mathrm{d}} & n & \Omega_{\mathrm{f}}
\end{array}\right)=\left(\begin{array}{lll}
1 & 1 & K \\
n & m & -(n+m)
\end{array}\right)\left(\begin{array}{ccc}
J_{\mathrm{i}} & J_{\mathrm{f}} & K \\
-\Omega_{\mathrm{i}} & \Omega_{\mathrm{f}} & n+m
\end{array}\right)
\end{aligned}
$$

and the normalization of the 3-j coefficients, equation 15 is obtained.

\section{Appendix C.}

INTEGRATION OVER KINETIC ENERGY. - The vibrational wavefunction of a molecule is simply given by :

$$
\left\langle R \mid \chi_{\Omega \varepsilon J}\right\rangle=R^{-1} u_{\Omega \varepsilon J}(R)
$$

where $u_{\Omega \varepsilon J}(R)$ is the solution of the Schrödinger equation :

$$
\frac{\hbar^{2}}{2 \mu} \frac{\mathrm{d}^{2}}{\mathrm{~d} R^{2}} u_{\Omega \varepsilon J}+\left[\varepsilon-V_{\Omega}(R)-\frac{\hbar^{2}}{2 \mu R^{2}}\left\{J(J+1)-\Omega^{2}\right\}\right] u_{\Omega \varepsilon J}=0
$$

For large $R$ values and energy $\varepsilon>0$, the asymptotic expression of $u_{\Omega \varepsilon J}$ is, with correct energy normalization, [35] :

$$
u_{\Omega \varepsilon J}(R)=\sqrt{\frac{2 \mu}{\pi \hbar^{2} K}} \sin \left(K R-\varphi_{\Omega \varepsilon J}\right)
$$


with

$$
\hbar^{2} K^{2} / 2 \mu=\varepsilon-V_{\Omega}(\infty) .
$$

The value of $\varphi$ is related to the well-known collision phase shift :

$$
\delta_{\Omega \Xi}=J \pi / 2-\varphi_{\Omega \Xi}
$$

$\varphi$ can also be related to $\alpha$, the classical angle of rotation of the internuclear axis during a " half-collision »

$$
\left(\frac{\partial \varphi_{\Omega \varepsilon J}}{\partial J}\right)_{\varepsilon}=\alpha
$$

In the expansion of the phase of the sine function in (C.3), the next term can be calculated in the W.K.B. approximation. Assuming that the long range behaviour of $V(R)$ is : $V(R)-C_{n} R^{-n}$, with $n \geqslant 2$,

with

$$
u_{\Omega \varepsilon J}(R) \rightarrow \sqrt{\frac{2 \mu}{\pi \hbar^{2} K}} \sin \phi_{\Omega \varepsilon}(R)
$$

$$
\phi_{\Omega \varepsilon J}(R)=K R-\varphi_{\Omega \varepsilon J}-\frac{J(J+1)-\Omega^{2}}{2 K R}+\mathcal{O}\left(\frac{1}{R^{2}}\right) .
$$

This new term is important to evaluate the minimum distance at which (C.3) is a good approximation of the wavefunction. We must calculate now the quantity $Z$ given by :

$$
Z=\int_{0}^{\infty} \mathrm{d} \varepsilon_{\mathrm{d}} \frac{\left\langle\chi_{\Omega_{\mathrm{i}} v_{\mathrm{J}} J_{1}} \mid \chi_{\Omega_{\mathrm{d}} \varepsilon_{\mathrm{d}} J_{\mathrm{d}}}\right\rangle\left\langle\chi_{\Omega_{\mathrm{d}} \varepsilon_{\mathrm{d}} J_{\mathrm{d}}} \mid \chi_{\Omega_{\mathrm{f}} \varepsilon_{\mathrm{f}} J_{\mathrm{f}}}\right\rangle}{E-\varepsilon_{\mathrm{d}}+i \Gamma_{\mathrm{d}}}
$$

Introducing the wavevectors $K_{\mathrm{d}}, K_{\mathrm{f}}$, associated to the levels and $|\mathrm{d}\rangle,|\mathrm{f}\rangle$ by (C.3b) and defining $K$ such that : $\hbar^{2} K^{2} / 2 \mu=E-V_{\Omega_{\mathrm{d}}}(\infty)$, we get :

$$
Z=\int_{0}^{\infty} \mathrm{d} K_{\mathrm{d}} f\left(K_{\mathrm{d}}\right) \int_{0}^{\infty} \mathrm{d} R \sin \left(K_{\mathrm{d}} R-\varphi_{\Omega_{\mathrm{d}} \varepsilon_{\mathrm{d}} J_{\mathrm{d}}}\right) \sin \left(K_{\mathrm{f}} R-\varphi_{\Omega_{\mathrm{f}} \varepsilon_{\mathrm{f}} J_{\mathrm{f}}}\right) /\left(K-K_{\mathrm{d}}+i \gamma_{\mathrm{d}}\right)
$$

with

$$
\gamma_{\mathrm{d}}=\frac{2 \mu \Gamma_{\mathrm{d}}}{\hbar^{2}\left(K+K_{\mathrm{d}}\right)}, \quad \text { and } \quad f\left(K_{\mathrm{d}}\right)=\frac{2 \mu \cdot\left\langle\chi_{\Omega_{\Omega_{1} v_{1} J_{\mathrm{i}}}} \mid \chi_{\Omega_{\mathrm{d} \varepsilon_{\mathrm{d}} J_{\mathrm{d}}}}\right\rangle}{K+K_{\mathrm{d}}^{2}}\left(\frac{K}{K_{\mathrm{f}}}\right)^{1 / 2}
$$

Asymptotic expressions of the wavefunctions have been used only for the term $\left\langle\chi_{\Omega_{\mathrm{d}} \varepsilon_{\mathrm{d}} J_{\mathrm{d}}} \mid \chi_{\Omega_{\mathrm{f}} \varepsilon_{\mathrm{f}} \mathrm{f}_{\mathrm{r}}}\right\rangle$ which is a slowly varying function of $K_{d}$; therefore integrating first on $K_{d}$, we obtain by usual complex plane integrating technique :

$$
\int_{0}^{\infty} \mathrm{d} K_{\mathrm{d}} f\left(K_{\mathrm{d}}\right) \frac{\sin \left(K_{\mathrm{d}} R-\varphi_{\Omega_{\mathrm{d}} \varepsilon_{\mathrm{d}} J_{\mathrm{d}}}\right)}{K-K_{\mathrm{d}}+i \gamma_{\mathrm{d}}} \simeq \pi f(K) \mathrm{e}^{i\left(K R-\varphi_{\left.\Omega_{\mathrm{d} \varepsilon_{\mathrm{d}} J_{\mathrm{d}}}\right)-\gamma_{\mathrm{d}}}\right.}
$$

This is a wavepacket which decays with increasing $R$. It is a striking image of the outgoing movement of the excited molecule decaying to the ground state by emission of radiation. Then the $R$ integration is straightforward. $Z$ is given by :

$$
Z=\frac{\mu}{\hbar^{2}} \frac{\left\langle\chi_{\Omega_{\mathrm{i}} V_{\mathrm{J}}} \mid \chi_{\Omega_{\mathrm{d}} \varepsilon_{\mathrm{d}} J_{\mathrm{d}}}\right\rangle}{\sqrt{K K_{\mathrm{f}}}\left[K-K_{\mathrm{f}}+i \gamma_{\mathrm{d}}\right]} \mathrm{e}^{i\left(\varphi \Omega_{\mathrm{f} \varepsilon \mathrm{f} J_{\mathrm{f}}}-\varphi_{\Omega_{\mathrm{d}} \varepsilon_{\mathrm{d}} J_{\mathrm{d}}}\right)}
$$

This quantity is important only near resonance, $K_{\mathrm{f}} \approx K$, and :

$$
Z \cong \frac{\left\langle\chi_{\Omega_{\mathrm{i}} V_{\mathrm{J}} \mathrm{J}_{\mathrm{i}}} \mid \chi_{\Omega_{\mathrm{d}} \varepsilon_{\mathrm{d}} J_{\mathrm{d}}}\right\rangle}{E-\left(\varepsilon_{\mathrm{f}}+E_{\mathrm{at}}\right)+i \Gamma_{\mathrm{d}}} \mathrm{e}^{i\left(\varphi_{\left.\Omega_{\mathrm{f}} \varepsilon_{\mathrm{f}} J_{\mathrm{f}}-\varphi_{\Omega_{\mathrm{d}} \varepsilon_{\mathrm{d}} J_{\mathrm{d}}}\right)}\right.}
$$

where $E_{\mathrm{at}}=V_{\Omega_{\mathrm{d}}}(\infty)-V_{\Omega_{\mathrm{f}}}(\infty)$ is the atomic excitation energy. It is now straightforward to obtain equation 16 from (C.10) assuming all other functions to be slowly varying, integration over $\varepsilon_{\mathrm{f}}$ of $\left\{\left[E-\left(\varepsilon_{\mathrm{f}}+E_{\mathrm{at}}\right)\right]^{2}+\Gamma_{\mathrm{d}}^{2}\right\}^{-\mathrm{i}}$ gives $\pi \Gamma_{\mathrm{d}}^{-1}$ and equation 16 is obtained. 
Before discussing the validity of(C.8),(C.9), we must point out the fact that the modulus of $Z$ is not affected by the relative phases $\varphi_{\Omega_{\mathrm{f}} \varepsilon_{\mathrm{f}} J_{\mathrm{f}}}$ and $\varphi_{\Omega_{\mathrm{d}} \varepsilon_{\mathrm{d}} J_{\mathrm{d}}}$. This is highly satisfactory because in the present problem of emission of radiation in the asymptotic region of internuclear distance, the second atom of the molecule acts only as a spectator. Its presence should not affect the intensity of emitted radiation i.e. the modulus of $Z$.

The validity of (C.8), (C.9), is good if the following conditions are fulfilled :

- the replacement of exact continuum wavefunctions by asymptotic formulae is justified only if the region in which these formulae are valid is large enough. Using (C.5), we see that the neglected term in the phase difference between $\chi_{\mathrm{d}}$ and $\chi_{\mathrm{f}}$ is :

$$
\frac{J_{\mathrm{f}}\left(J_{\mathrm{f}}+1\right)}{2 K_{\mathrm{f}} R}-\frac{J_{\mathrm{d}}\left(J_{\mathrm{d}}+1\right)}{2 K_{\mathrm{d}} R}
$$

whose modulus is usually less than $2 J / K R$ (near resonance $K_{\mathrm{f}} \sim K_{\mathrm{d}} \sim K$ ) because $\left|J_{\mathrm{d}}-J_{\mathrm{f}}\right| \leqslant 1$. This phase difference is less than a specified value (say $10^{-2}$ radians) for :

$$
R_{0}(\AA) \geqslant 20 \mathrm{~J}
$$

( $K$ is taken equal to $10 \AA^{-1}$, corresponding for $\mathrm{Ca}_{2}$ to a kinetic recoil energy $\hbar^{2} K^{2} / 2 \mu \sim 100 \mathrm{~cm}^{-1}$ ). We guess that this lower limit is underestimated for a low $J$ value because potential terms in $C_{n} R^{-n}$ dominate the centrifugal terms, but for a typical $J$ value for $\mathrm{Ca}_{2}(J=50)$, this value is :

$$
R_{0} \geqslant 1000 \AA \text {. }
$$

For the same value of $K$, the wavepacket in the excited state has a characteristic length for $\mathrm{Ca}_{2}$

$$
L=1 / \gamma_{\mathrm{d}} \sim 16000 \AA \text {. }
$$

Therefore the asymptotic region extends over a sufficiently large region to justify the validity of the present calculation. If the kinetic energy increases, $R$ decreases and $L$ increases, and the accuracy of the calculation increases.

\section{Appendix D.}

STUDY OF THE MATRIX ELEMENTS OF $T(E)$. - In order to calculate the cross-section defined by equation 4 , we need the matrix elements $\left\langle\alpha_{\mathrm{i}} ; \mathbf{k}_{\mathrm{i}} \mathbf{e}_{\mathrm{i}}|T(E)| \alpha_{\mathrm{f}} ; \mathbf{k}_{\mathrm{f}} \mathbf{e}_{\mathrm{f}}\right\rangle$, which, using equation 5 , can be written :

$$
\begin{aligned}
\left\langle\alpha_{\mathrm{i}} ; \mathbf{k}_{\mathrm{i}} \mathbf{e}_{\mathrm{i}}|T(E)| \alpha_{\mathrm{f}} ; \mathbf{k}_{\mathrm{f}} \mathbf{e}_{\mathrm{f}}\right\rangle=\sum_{\alpha_{\mathrm{d} \alpha} \alpha_{\mathrm{d}}^{\prime}}\left\langle\alpha_{\mathrm{i}} ; \mathbf{k}_{\mathrm{i}} \mathbf{e}_{\mathrm{i}}\left|H_{\mathrm{int}}\right| \alpha_{\mathrm{d}} ; 0\right\rangle \times & \\
& \times\left\langle\alpha_{\mathrm{d}} ; 0\left|G^{+}(E)\right| \alpha_{\mathrm{d}}^{\prime} ; 0\right\rangle\left\langle\alpha_{\mathrm{d}}^{\prime} ; 0\left|H_{\text {int }}\right| \alpha_{\mathrm{f}} ; \mathbf{k}_{\mathrm{f}} \mathbf{e}_{\mathrm{f}}\right\rangle .
\end{aligned}
$$

We need now the explicit form of the matrix elements of $G^{+}(E)$ which is the resolvent operator defined in equation 6. Defining the projection operators :

$$
P=\sum_{\alpha_{\mathrm{d}}}\left|\alpha_{\mathrm{d}} ; 0\right\rangle\left\langle\alpha_{\mathrm{d}} ; 0\right| ; \quad Q=1-P
$$

it can be shown [18] :

$$
P G^{+}(E) P=\left[E-H_{0}-P R\left(E^{+}\right) P\right]^{-1}
$$

where $R\left(E^{+}\right)$is the level-width operator :

$$
R\left(E^{+}\right)=H_{\mathrm{int}}+H_{\mathrm{int}}\left(E^{+}-Q H Q\right)^{-1} H_{\mathrm{int}}
$$

with $E^{+}=\lim _{\eta \rightarrow 0^{+}}(E+i \eta)$. We are thus interested in the matrix elements of $R\left(E^{+}\right)$in the $P$ subspace. It is straightforward to show that $R\left(E^{+}\right)$is diagonal with respect to $J_{\mathrm{d}}$ and $M_{\mathrm{d}}$. From (D.4), we have :

$$
\begin{aligned}
\left\langle\Omega_{\mathrm{d}} \varepsilon_{\mathrm{d}} J_{\mathrm{d}} M_{\mathrm{d}} ; 0\left|R\left(E^{+}\right)\right| \Omega_{\mathrm{d}}^{\prime} \varepsilon_{\mathrm{d}}^{\prime} J_{\mathrm{d}}^{\prime} M_{\mathrm{d}}^{\prime} ; 0\right\rangle=\sum_{\Omega \varepsilon M} \sum_{\mathbf{k e}}\left\langle\Omega_{\mathrm{d}} \varepsilon_{\mathrm{d}} J_{\mathrm{d}} M_{\mathrm{d}} ; 0\left|H_{\mathrm{int}}\right| \Omega \varepsilon J M ; \mathbf{k e}\right\rangle \\
{[E-\varepsilon-\hbar c k+i \eta]^{-1}\left\langle\Omega \varepsilon J M ; \mathbf{k e}\left|H_{\mathrm{int}}\right| \Omega_{\mathrm{d}}^{\prime} \varepsilon_{\mathrm{d}}^{\prime} J_{\mathrm{d}}^{\prime} M_{\mathrm{d}}^{\prime} ; 0\right\rangle }
\end{aligned}
$$


where the sum is over all possible final states. Using equations 8 and 11, we get :

$$
\begin{aligned}
& \left\langle\Omega_{\mathrm{d}} \varepsilon_{\mathrm{d}} J_{\mathrm{d}} M_{\mathrm{d}} ; 0\left|R\left(E^{+}\right)\right| \Omega_{\mathrm{d}}^{\prime} \varepsilon_{\mathrm{d}}^{\prime} J_{\mathrm{d}}^{\prime} M_{\mathrm{d}}^{\prime} ; 0\right\rangle=\sum_{\Omega \in M} \sum_{\mathbf{k e}} \hbar c k \times \\
& \times[E-\varepsilon-\hbar c k+i \eta]^{-1} \sum_{p, q}(-1)^{p}(e)_{p}^{1}\left\langle\Omega_{\mathrm{d}} \varepsilon_{\mathrm{d}} J_{\mathrm{d}}\left|(D)_{m}^{1}\right| \Omega \varepsilon J\right\rangle \\
& \times\left(2 J_{\mathrm{d}}+1\right)^{1 / 2}(2 J+1)^{1 / 2}(-1)^{J_{\mathrm{d}}-M_{\mathrm{d}}}\left(\begin{array}{crc}
J_{\mathrm{d}} & 1 & J \\
-M_{\mathrm{d}} & -p & M
\end{array}\right)(-1)^{J_{\mathrm{d}}-\Omega_{\mathrm{d}}} \\
& \times\left(\begin{array}{rcc}
J_{\mathrm{d}} & 1 & J \\
-\Omega_{\mathrm{d}} & m & \Omega
\end{array}\right)(-1)^{q}\left(e^{*}\right)_{q}^{1}\left\langle\Omega \varepsilon J\left|(D)_{n}^{1}\right| \Omega_{\mathrm{d}}^{\prime} \varepsilon_{\mathrm{d}}^{\prime} J_{\mathrm{d}}^{\prime}\right\rangle(2 J+1)^{1 / 2} \\
& \times\left(2 J_{\mathrm{d}}^{\prime}+1\right)^{1 / 2}(-1)^{J-M}\left(\begin{array}{crc}
J & 1 & J_{\mathrm{d}}^{\prime} \\
-M & -q & M_{\mathrm{d}}^{\prime}
\end{array}\right)(-1)^{J-\Omega}\left(\begin{array}{ccc}
J & 1 & J_{\mathrm{d}}^{\prime} \\
-\Omega & n & \Omega_{\mathrm{d}}^{\prime}
\end{array}\right) \\
& m=\Omega_{\mathrm{d}}-\Omega ; \quad n=-\Omega_{\mathrm{d}}^{\prime}+\Omega \text {. }
\end{aligned}
$$

Since we sum over all possible final polarization $\mathbf{e}$, we have :

$$
(e)_{p}^{1}\left(e^{*}\right)_{q}^{1}=(-1)^{p} \delta_{-p q},
$$

and the sum over $M$ and $p$ can be performed by the use of the normalization of the $3-j$ coefficients. The result is :

$$
\begin{aligned}
\left\langle\Omega_{\mathrm{d}} \varepsilon_{\mathrm{d}} J_{\mathrm{d}} M_{\mathrm{d}} ; 0\left|R\left(E^{+}\right)\right| \Omega_{\mathrm{d}}^{\prime}\right. & \varepsilon_{\mathrm{d}}^{\prime} \\
& \left.J_{\mathrm{d}}^{\prime} M_{\mathrm{d}}^{\prime} ; 0\right\rangle=\sum_{\Omega \varepsilon j k} \hbar c k \times \\
& \times[E-\varepsilon-\hbar c k+i \eta]^{-1}\left\langle\Omega_{\mathrm{d}} \varepsilon_{\mathrm{d}} J_{\mathrm{d}}\left|(D)_{m}^{1}\right| \Omega \varepsilon J\right\rangle \\
& \times\left\langle\Omega \varepsilon J\left|(D)_{n}^{1}\right| \Omega_{\mathrm{d}}^{\prime} \varepsilon_{\mathrm{d}}^{\prime} J_{\mathrm{d}}\right\rangle(2 J+1)(-1)^{-2 M_{\mathrm{d}}-\Omega-\Omega_{\mathrm{d}}} \\
& \times\left(\begin{array}{rrrr}
J_{\mathrm{d}} & 1 & J \\
-\Omega_{\mathrm{d}} & m & \Omega
\end{array}\right)\left(\begin{array}{rrr}
J & 1 & J_{\mathrm{d}} \\
-\Omega & n & \Omega_{\mathrm{d}}^{\prime}
\end{array}\right) \delta_{J_{\mathrm{d}} J_{\mathrm{d}}^{\prime}}, \delta_{M_{\mathrm{d}} M_{\mathrm{d}}^{\prime}} \\
& \quad m=\Omega_{\mathrm{d}}-\Omega ; \quad n=-\Omega_{\mathrm{d}}^{\prime}+\Omega .
\end{aligned}
$$

In this work, we have assumed that dissociation is fast with respect to fluorescence. We thus invoke the Franck-Condon approximation, equation 15 , and the continuum wavefunctions are replaced by their asymptotic form. Using the results of appendix $\mathrm{C}$, the $J$-dependence on the product

$$
\left\langle\Omega_{\mathrm{d}} \varepsilon_{\mathrm{d}} J_{\mathrm{d}}\left|(D)_{m}^{1}\right| \Omega \varepsilon J\right\rangle\left\langle\Omega \varepsilon J\left|(D)_{n}^{1}\right| \Omega_{\mathrm{d}}^{\prime} \varepsilon_{\mathrm{d}}^{\prime} J_{\mathrm{d}}\right\rangle
$$

disappears and the sum over $J$ can be performed by the use of the normalization relations of the 3-j coefficients. The final result is :

$$
\left\langle\Omega_{\mathrm{d}} \varepsilon_{\mathrm{d}} J_{\mathrm{d}} M_{\mathrm{d}} ; 0\left|R\left(E^{+}\right)\right| \Omega_{\mathrm{d}}^{\prime} \varepsilon_{\mathrm{d}}^{\prime} J_{\mathrm{d}}^{\prime} M_{\mathrm{d}}^{\prime} ; 0\right\rangle=\delta\left(\varepsilon_{\mathrm{d}}-\varepsilon_{\mathrm{d}}^{\prime}\right) \delta_{\Omega_{\mathrm{d}} \Omega_{\mathrm{d}}^{\prime}} \delta_{J_{\mathrm{d}} J_{\mathrm{d}}^{\prime}} \delta_{M_{\mathrm{d}} M_{\mathrm{d}}^{\prime}}\left(i \Gamma_{\mathrm{d}}\right)
$$

where $\Gamma_{\mathrm{d}}$ is the atomic fluorescence linewidth. From (D.8) and (D.9) it is now straightforward to write equation 7.

\section{Appendix E.}

RELATIONSHIP BETWEEN PHOTOFRAGMENTS POLARIZATION AND ANGULAR DISTRIBUTIONS. - From equation 7 we can write

$$
\frac{\mathrm{d} \sigma}{\mathrm{d} \hat{\omega}_{\mathrm{f}}} \propto \sum_{\alpha_{\mathrm{f}}} \sum_{\alpha_{\mathrm{d}}} \sum_{\alpha_{\mathrm{d}}^{\prime}}\left\langle\alpha_{\mathrm{i}}\right| \text { D. } \mathbf{e}_{\mathrm{i}}\left|\alpha_{\mathrm{d}}\right\rangle\left\langle\alpha_{\mathrm{d}}\right| \text { D. } \mathrm{e}_{\mathrm{f}}^{*}\left|\alpha_{\mathrm{f}}\right\rangle\left\langle\alpha_{\mathrm{d}}^{\prime}\right|\left(\text { D. } \mathbf{e}_{\mathrm{i}}\right)^{\dagger}\left|\alpha_{\mathrm{i}}\right\rangle\left\langle\alpha_{\mathrm{f}}\right|\left(\text { D. }_{\mathrm{f}}^{*}\right)^{\dagger}\left|\alpha_{\mathrm{d}}^{\prime}\right\rangle .
$$

We now introduce the plane-waves

$$
\left|\Omega_{\mathrm{d}} ; \mathbf{K}_{\mathrm{d}}\right\rangle=\sum_{J_{\mathrm{d}} M_{\mathrm{d}}}\left(2 J_{\mathrm{d}}+1\right)^{1 / 2} \mathrm{e}^{-i \varphi_{\Omega_{\mathrm{d}} \varepsilon_{\mathrm{d}} J_{\mathrm{d}}}} i^{J_{\mathrm{d}}} \mathfrak{D}_{M_{\mathrm{d}} \Omega_{\mathrm{d}}}^{J_{*}^{*}}(\varphi, \theta, 0)\left|\Omega_{\mathrm{d}} \varepsilon_{\mathrm{d}} J_{\mathrm{d}} M_{\mathrm{d}}\right\rangle
$$

where $\left|\Omega_{\mathrm{d}} \varepsilon_{\mathrm{d}} J_{\mathrm{d}} M_{\mathrm{d}}\right\rangle$ are the spherical waves defined by equation 9 , and $\mathfrak{D}_{M_{\mathrm{d}} \Omega_{\mathrm{d}}}^{J_{\mathrm{d}}^{*}}(\varphi, \theta, 0)$ are the Wigner functions 
depending on the polar angles $\theta, \varphi$ defining the direction of $\mathbf{K}_{\mathbf{d}}$ with respect to the laboratory frame. Introducing (E.2) into (E.1), and using equations 8 and 11 , we get

$$
\begin{aligned}
& \frac{\mathrm{d} \sigma}{\mathrm{d} \hat{\omega}_{\mathrm{f}}} \propto \sum_{\alpha_{\mathrm{f}}} \sum_{\alpha_{\mathrm{d}}^{\prime}} \sum_{\Omega_{\mathrm{d}} J_{\mathrm{d}} M_{\mathrm{d}}} \sum_{\tilde{J}_{\mathrm{d}} \hat{M}_{\mathrm{d}}} \int \mathrm{d} \hat{\omega} \mathrm{e}^{-i \varphi_{\Omega_{\mathrm{d}} \varepsilon_{\mathrm{d}} J_{\mathrm{d}}} i^{J_{\mathrm{d}}} \times} \\
& \times \mathrm{e}^{i \varphi_{\Omega_{\mathrm{d}} \theta_{\mathrm{d}} \tilde{\mathrm{d}}} i^{\tilde{J_{\mathrm{d}}}}}\left\langle\alpha_{\mathrm{i}}\left|\mathbf{D} . \mathrm{e}_{\mathrm{i}}\right| \Omega_{\mathrm{d}} \varepsilon_{\mathrm{d}} J_{\mathrm{d}} M_{\mathrm{d}}\right\rangle \mathscr{D}_{M_{\mathrm{d}} \Omega_{\mathrm{d}}}^{s_{\mathrm{d}}^{*}}(\varphi, \theta, 0) \\
& \times\left\langle\Omega_{\mathrm{d}} \varepsilon_{\mathrm{d}} \widetilde{J}_{\mathrm{d}} \tilde{M}_{\mathrm{d}}\right| \text { D. } \mathrm{e}_{\mathrm{f}}^{*}\left|\alpha_{\mathrm{f}}\right\rangle \mathfrak{D}_{\hat{M}_{\mathrm{d}} \Omega_{\mathrm{d}}}^{\tilde{J}_{\mathrm{d}}}(\varphi, \theta, 0)\left\langle\alpha_{\mathrm{d}}^{\prime}\left|\left(\mathbf{D} . \mathrm{e}_{\mathrm{i}}\right)^{\dagger}\right| \alpha_{\mathrm{i}}\right\rangle\left\langle\alpha_{\mathrm{f}}\left|\left(\mathbf{D} . \mathrm{e}_{\mathrm{f}}^{*}\right)^{\dagger}\right| \alpha_{\mathrm{d}}^{\prime}\right\rangle \text {. }
\end{aligned}
$$

In the axial recoil approximation $\mathrm{e}^{-i \varphi_{\Omega_{\mathrm{d}} \varepsilon_{\mathrm{d}} J_{\mathrm{d}}}} i^{J_{\mathrm{d}}}=1$ and the sum over $J_{\mathrm{d}}$ and $M_{\mathrm{d}}$ can be performed with the result :

$$
\sum_{J_{\mathrm{d}} M_{\mathrm{d}}}\left\langle\alpha_{\mathrm{i}}\right| \text { D.e } \mathbf{e}_{\mathrm{i}}\left|\Omega_{\mathrm{d}} \varepsilon_{\mathrm{d}} J_{\mathrm{d}} M_{\mathrm{d}}\right\rangle \mathcal{D}_{M_{\mathrm{d}} \Omega_{\mathrm{d}}}^{J_{*}^{*}}(\varphi, \theta, 0)=\sum_{p, m}(-1)^{M_{\mathrm{i}}+\Omega_{\mathrm{i}}}\left\langle\Omega_{\mathrm{i}}\left|(D)_{m}^{1}\right| \Omega_{\mathrm{d}}\right\rangle\left(e_{\mathrm{i}}\right)_{p}^{1} \mathcal{D}_{M_{\mathrm{i}} \Omega_{\mathrm{i}}}^{J_{\mathrm{i}}^{*}}(\varphi, \theta, 0) .
$$

The sum over $J_{\mathrm{d}}$ and $M_{\mathrm{d}}$ can be performed exactly in the same way, and finally, we get :

$$
\begin{aligned}
\frac{\mathrm{d} \sigma}{\mathrm{d} \hat{\omega_{\mathrm{f}}}} \propto \sum_{\alpha_{\mathrm{f}}} \sum_{x_{\mathrm{d}}^{\prime}} \sum_{\Omega_{\mathrm{d}}} \int \mathrm{d} \hat{\omega} \mathcal{D}_{M_{\mathrm{i}} \Omega_{\mathrm{i}}}^{J_{\mathrm{i}}^{*}}(\varphi, \theta, 0) g_{\Omega_{\mathrm{i}} \Omega_{\mathrm{d}}}(\theta, \varphi) \\
\mathcal{D}_{M_{\mathrm{f}} \Omega_{\mathrm{f}}}^{J_{\mathrm{f}}^{*}}(\varphi, \theta, 0) a_{\Omega_{\mathrm{d}} \Omega_{\mathrm{f}}}(\theta, \varphi)\left\langle\alpha_{\mathrm{d}}^{\prime}\left|\left(\mathbf{D} \cdot \mathbf{e}_{\mathrm{i}}\right)^{\dagger}\right| \alpha_{\mathrm{i}}\right\rangle\left\langle\alpha_{\mathrm{f}}\left|\left(\mathbf{D} . \mathbf{e}_{\mathrm{f}}^{*}\right)^{\dagger}\right| \alpha_{\mathrm{d}}^{\prime}\right\rangle
\end{aligned}
$$

where the functions $g_{\Omega_{\mathrm{i}} \Omega_{\mathrm{d}}}(\theta, \varphi)$ and $a_{\Omega_{\mathrm{d}} \Omega_{\mathrm{f}}}(\theta, \varphi)$ are defined by equations 35 . The sums over $\alpha_{\mathrm{f}}$ and $\alpha_{\mathrm{d}}^{\prime}$ imply sums over $J_{\mathrm{f}} M_{\mathrm{f}}$ and $J_{\mathrm{d}}^{\prime} M_{\mathrm{d}}^{\prime}$ which can again be performed by the use of (E.4). We finally get :

$$
\frac{\mathrm{d} \sigma}{\mathrm{d} \hat{\omega}_{\mathrm{f}}} \propto \sum_{\Omega_{\mathrm{d}}} \sum_{\Omega_{\mathrm{d}}^{\prime}} \int \mathrm{d} \hat{\omega} \mathfrak{D}_{M_{\mathrm{i}} \Omega_{\mathrm{i}}}^{J_{t}^{*}}(\varphi, \theta, 0) \mathfrak{D}_{M_{\mathrm{i}} \Omega_{\mathrm{i}}}^{J_{\mathrm{i}}}(\varphi, \theta, 0) g_{\Omega_{\mathrm{i}} \Omega_{\mathrm{d}}}(\theta, \varphi) a_{\Omega_{\mathrm{d}} \Omega_{\mathrm{f}}}(\theta, \varphi) g_{\Omega_{\mathrm{i}} \Omega_{\mathrm{d}}^{\prime}}^{*}(\theta, \varphi) a_{\Omega_{\mathrm{d}} \Omega_{\mathrm{f}}}^{*}(\theta, \varphi) .
$$

Now, averaging this expression over $M_{\mathrm{i}}$ (the molecule is randomly oriented), and using the normalization of the Wigner functions, we obtain equation 34.

\section{References}

[1] For a review see Simons, J. P., in : Gas Kinetics and Energy Transfer 2 (Chem. Soc. Spec. Period. Rep., London) 1977, p. 56.

[2] Poliakoff, E. D., Dehmer, J. L., Dill, D., Parr, A. C., JACKSON, K. H. and ZARE, R. N., Phys. Rev. Lett. 46 (1981) 907.

[3] Prisant, M. G., Rettner, C. T. and Zare, R. N., J. Chem. Phys. 75 (1981) 2222.

[4] Van Brunt, R. J. and Zare, R. N., J. Chem. Phys. 48 (1968) 4304.

[5] ZARE, R. N., Ph. D. Thesis, Harvard University (1964); Mol. Photochem. 4 (1972) 1. See also

YANG, S. C. and Bersohn, R., J. Chem. Phys. 61 (1974) 4400.

[6] Bersohn, R. and Lin, S. H., Adv. Chem. Phys. 16 (1969) 67.

[7] Stepanov, B. I. and Gribkovskit, V. P., Theory of Luminescence (Ilife Books Ltd., London) 1968.

[8] a) Chamberlain, G. A. and Simons, J. P., Chem. Phys. Lett. 32 (1975) 355 ; J. Chem. Soc. Faraday II 71 (1975) 2043;

b) Mac Pherson, M. J., Simon, J. P. and Zare, R. N., Molec. Phys. 38 (1979) 2049;

c) Loge, G. W. and ZARe, R. N., Molec. Phys. 43 (1981) 1419 ;

d) Husain, J., Wiesenfeld, J. R. and Zare, R. N., J. Chem. Phys. 72 (1980) 2479.

e) Loge, G. W. and Wiesenfeld, J. R., Chem. Phys. Lett. 78 (1981) 32.
[9] Rothe, E. W., Krause, U. and Düren, R., Chem. Phys. Lett. 72 (1980) 100.

[10] Vigute, J., Grangier, P., Roger, G. and Aspect, A., J. Physique Lett. 42 (1981) L-531.

[11] VASYUTINSKIII, O. S., JETP Lett. 31 (1980) 428.

[12] VASYUTINSKIĨ, O. S., JETP 54 (1981) 855.

[13] Hougen, J. T., The Calculation of Rotational Energy Levels and Rotational Line Intensities in Diatomic Molecules (NBS Monograph) 1970.

[14] Zare, R. N., J. Chem. Phys. 45 (1966) 4510.

[15] Goundard, G. and Lehmann, J. C., J. Physique 34 (1973) 693 ;

Broyer, M., Gouedard, G., Lehmann, J. C. and Viguk, J., Adv. Atom. Mol. Phys. 12 (1976) 165.

[16] ViguE, J., Thesis, Paris (1978), unpublished.

[17] Edmonds, A. R., in Angular Momentum in Quantum Mechanics (Princeton University Press) 1957.

[18] Beswick, J. A. and DuruP, J., in Chemical Photophysics (Editions du C.N.R.S.) 1979.

[19] Jonah, C., J. Chem. Phys. 55 (1971) 1915.

[20] Jones, R. O., J. Chem. Phys. 71 (1979) 1300.

[21] Stevens, W. J. and Krauss, M., J. Chem. Phys. 67 (1977) 1977.

[22] JefFrey Hay, P., DunNing, Jr., T. H. and RAFFeNetti, R. C., J. Chem. Phys. 65 (1976) 2679.

[23] Balfour, W. J. and Whitlock, R. F., Can. J. Phys. 72 (1980) 1864.

[24] Vidal, C. R., J. Chem. Phys. 72 (1980) 1864. 
[25] Katsumi Sakurai and Broida, H. P., J. Chem. Phys. 65 (1976) 1138 ;

Wyss, J. C., J. Chem. Phys. 71 (1979) 2949.

[26] Andrews, L., Duley, W. W. and Brewer, L., J. Mol. Spectrosc. 70 (1978) 41.

Bondybey, V. E. and Albiston, C., J. Chem. Phys. 68 (1978) 3172.

Miller, J. C. and Andrews, L., J. Chem. Phys. 69 (1978) 2054.

[27] Mrller, J. C., Mowery, R. L., Krausz, E. R., JaCobs, S. M., KIM, H. W., SchATZ, P. N. and ANDrews, L., J. Chem. Phys. 74 (1981) 6349.

[28] Moore, C., in Atomic Energy Levels (NBS 467) 1949.

[29] King, G. W. and van Vleck, J. H., Phys. Rev. 55 (1939) 1165.

[30] Atomic Transition Probabilities, vol. II (NSRDS/NBS 22) 1969.

[31] Aspect, A., Imbert, C. and Roger, C., Opt. Commun. 34 (1980) 46.

[32] Zinsli, P. E., J. Phys. E 11 (1978) 17.
[33] ChILD, M. S., J. Mol. Spectrosc. 33 (1970) 487. Vigué, J., Ann. Phys. 3 (1982) 155.

[34] Landau, L. and LifChitz, E., in Mechanics (Pergamon Press, Oxford) 1960.

[35] Messiah, A., in Mécanique Quantique (Dunod Editeur, Paris) 1962.

[36] Cohen Tannoudj, C. and Avan, P., Colloque International du C.N.R.S. $\mathrm{n}^{\circ}$ 273, p. 93, Editions du C.N.R.S., Paris (1977).

[37] Sinha, M. P., Caldwell, C. D. and Zare, R. N., J. Chem. Phys. 61 (1974) 491.

[38] Band, Y. B. and Freed, K. E., Chem. Phys. Lett. 79 (1981) 238.

Band, Y. B. and Freed, K. F., Chem. Phys. Lett. 79 (1981) 233.

[39] Grosser, J., J. Phys. B 14 (1981) 1449.

[40] Singer, S. J. and FREED, K. F., private communication (September 1982).

[41] Child, M. S., Molecular Collision Theory (Academic Press) 1974. 